\title{
REGULARITY OF INTERFACES FOR A PUCCI TYPE SEGREGATION PROBLEM
}

\author{
L. CAFFARELLI, S. PATRIZI, V. QUITALO, AND M. TORRES
}

\begin{abstract}
We show the existence of a Lipschitz viscosity solution $u$ in $\Omega$ to a system of fully nonlinear equations involving Pucci-type operators. We study the regularity of the interface $\partial\{u>0\} \cap \Omega$ and we show that the viscosity inequalities of the system imply, in the weak sense, the free boundary condition $u_{\nu_{+}}^{+}=u_{\nu_{-}}^{-}$, and hence $u$ is a solution to a two-phase free boundary problem. We show that we can apply the classical method of sup-convolutions developed by the first author in 5, 6, and generalized by Wang 20,21 and Feldman 11 to fully nonlinear operators, to conclude that the regular points in $\partial\{u>0\} \cap \Omega$ form an open set of class $C^{1, \alpha}$. A novelty in our problem is that we have different operators, $\mathcal{F}^{+}$and $\mathcal{F}^{-}$, on each side of the free boundary. In the particular case when these operators are the Pucci's extremal operators $\mathcal{M}^{+}$and $\mathcal{M}^{-}$, our results provide an alternative approach to obtain the stationary limit of a segregation model of populations with nonlinear diffusion in 19.
\end{abstract}

\section{INTRODUCTION}

The work in the present paper is motivated by the study of the regularity of the free boundary for a limit problem obtained from a segregation model with nonlinear diffusion studied by the third author in [19]. In the case of two populations, the model takes the form

$$
\left\{\begin{array}{l}
\mathcal{M}^{-}\left(u_{1}^{\epsilon}\right)=\frac{1}{\epsilon} u_{1}^{\epsilon} u_{2}^{\epsilon} \quad \text { in } \Omega \\
\mathcal{M}^{-}\left(u_{2}^{\epsilon}\right)=\frac{1}{\epsilon} u_{1}^{\epsilon} u_{2}^{\epsilon} \quad \text { in } \Omega \\
u_{i}^{\epsilon}=f_{i} \quad i=1,2, \quad \text { on } \partial \Omega
\end{array}\right.
$$

where $\Omega$ is a bounded Lipschitz domain of $\mathbb{R}^{n}, f_{1}$ and $f_{2}$ are non-negative, non-zero, Hölder continuous function defined on $\partial \Omega$, with disjoint supports, $\mathcal{M}^{-}$denotes the negative Pucci's extremal operator that will be described later. The non-negative solution $u_{i}^{\epsilon}, i=1,2$ of (1.1) can be seen as a density of the population $i$, and the parameter $\frac{1}{\epsilon}>0$ characterizes the level of competition between species. In [19] it is proven that along a subsequence, $u_{1}^{\epsilon}$ and $u_{2}^{\epsilon}$ converge uniformly in $\Omega$, as $\epsilon \rightarrow 0^{+}$, respectively to $u_{1}$ and $u_{2}$, non-negative locally Lipschitz functions,

2010 Mathematics Subject Classification. Primary: 35J60; Secondary: 35R35, 35B65, 35Q92.

Key words and phrases. Fully nonlinear elliptic systems, Pucci operators, Regularity for viscosity solutions, Segregation of populations, Regularity of the free boundary.

This work was partially supported by the Centre for Mathematics of the University of Coimbra UID/MAT/00324/2013, funded by the Portuguese Government through FCT/MEC and co-funded by the European Regional Development Fund through the Partnership Agreement PT2020. 
solutions of the following free boundary problem, for $i, j=1,2$,

$$
\begin{cases}\mathcal{M}^{-}\left(u_{i}\right)=0 & \text { in }\left\{u_{i}>0\right\} \\ \mathcal{M}^{-}\left(u_{i}-u_{j}\right) \leq 0 & \text { in } \Omega \\ u_{1} u_{2}=0 & \text { in } \Omega \\ u_{i}=f_{i} & \text { on } \partial \Omega .\end{cases}
$$

Let $u:=u_{1}-u_{2}$, then $u_{1}=u^{+}, u_{2}=u^{-}$, where $u^{+}, u^{-}$are respectively the positive and negative part of $u$, and system (1.2) can be rewritten in terms of $u$ as follows

$$
\begin{cases}\mathcal{M}^{-}(u)=0 & \text { in }\{u>0\} \\ \mathcal{M}^{+}(u)=0 & \text { in }\{u<0\} \\ \mathcal{M}^{-}(u) \leq 0 & \text { in } \Omega \\ \mathcal{M}^{+}(u) \geq 0 & \text { in } \Omega \\ u=f & \text { on } \partial \Omega,\end{cases}
$$

where $f=f_{1}-f_{2}$ and $\mathcal{M}^{+}(u)=-\mathcal{M}^{-}(-u)$ is the positive Pucci's operator.

In the present paper we study problems likewise (1.3) in a more general setting. Precisely, we consider the following free boundary problem,

$$
\begin{cases}\mathcal{F}^{-}(u)=0 & \text { in }\{u>0\} \\ \mathcal{F}^{+}(u)=0 & \text { in }\{u<0\} \\ \mathcal{F}^{-}(u) \leq 0 & \text { in } \Omega \\ \mathcal{F}^{+}(u) \geq 0 & \text { in } \Omega \\ u=f & \text { on } \partial \Omega\end{cases}
$$

in a bounded smooth domain $\Omega \subset \mathbb{R}^{n}$, where $f$ is a Lipschitz function defined on $\partial \Omega$ and $\mathcal{F}^{-}$and $\mathcal{F}^{+}$are uniformly elliptic operators belonging to a class of extremal operators that includes the Pucci's operators $\mathcal{M}^{-}$and $\mathcal{M}^{+}$. Therefore the limit problem (1.3) can be seen as a particular case of (1.4).

We first prove the existence of a Lipschitz solution $u$ of (1.4). Then, we study the regularity of the free boundary set

$$
\Gamma:=\partial\{u>0\} \cap \Omega .
$$

Denote $u_{1}=u^{+}$and $u_{2}=u^{-}$, and let $\nu_{i}$ be the interior unit normal vector to $\left\{u_{i}>0\right\}$. At this stage we have no information about the regularity of the free boundary $\Gamma$ and the vectors $\nu_{i}$ may not be defined at every point of $\Gamma$. However, we can prove that any Lipschitz solution of (1.4) satisfies in a weak sense (viscosity sense) the following free boundary condition

$$
\frac{\partial u_{1}}{\partial \nu_{1}}=\frac{\partial u_{2}}{\partial \nu_{2}} \quad \text { on } \Gamma
$$

that is, the normal derivative of $u$ is continuous across the free boundary.

This will allow us to apply the regularity theory developed by Caffarelli in the papers [4-6] for free boundary problems associated to linear operators and then extended by Wang [20,21] to the case of fully nonlinear uniformly elliptic concave operators, to show that the subset of regular points of the free boundary is relatively open in $\Gamma$ and locally of class $C^{1, \alpha}, 0<\alpha \leq 1$.

Let us describe more in details the results of the present paper and the strategies followed. Let $x_{0} \in \Gamma$ and assume that $\Gamma$ is smooth around $x_{0}$, then since $u$ is a viscosity solution of the 
first and second equation in (1.4), by the Hopf Lemma we have

$$
0<\frac{\partial u_{1}}{\partial \nu_{1}}\left(x_{0}\right), \frac{\partial u_{2}}{\partial \nu_{2}}\left(x_{0}\right)<+\infty
$$

that is $u$ has linear growth away from the free boundary around $x_{0}$. Thus, we expect that at points where the solution $u$ "behaves well", in fact both $u_{1}$ and $u_{2}$ have locally linear growth away from the free boundary. The linear behavior of $u_{i}$ at a point $x_{0}$ of the free boundary without regularity assumptions on $\Gamma$ can be defined as follows: there exists $\tilde{r}=\tilde{r}\left(x_{0}\right)>0$ and $M=M\left(x_{0}\right)>0$ such that for any $0<r<\tilde{r}$,

$$
\sup _{B_{r}\left(x_{0}\right)} u_{i} \geq M r
$$

A barrier argument shows that the function $u_{i}$ satisfies (1.5) at points of $\Gamma$ where there is a tangent ball to $\Gamma$ contained in its support, as we will see. Points with this property are dense in $\Gamma$. Thus, we define $x_{0} \in \Gamma$ to be regular if (1.5) holds true for at least one among $u_{1}$ and $u_{2}$, see Definition 6.1. Then by using that $u$ satisfies in the viscosity sense

$$
\mathcal{F}^{-}(u) \leq 0 \leq \mathcal{F}^{+}(u) \text { in } \Omega
$$

we can actually prove that both $u_{1}$ and $u_{2}$ have linear behavior at any regular point, as expected. The viscosity inequalities (1.6) have to be understood as a sort of free boundary conditions since they are satisfied in the whole $\Omega$ and thus across the free boundary too.

Now, solutions of (1.4) have the properties that the positive and negative parts are subharmonic in $\Omega$. Therefore, we can perform a blow up analysis by using the monotonicity formula. In particular, we can show that if $u$ is a Lipschitz solution of (1.4), then around any regular point the free boundary is flat, meaning that it can be trapped in a narrow neighborhood in between two Lipschitz graphs. If in addition there is a tangent ball from one side at $x_{0} \in \Gamma$, meaning that the ball is contained either in the positivity set of $u$ or in its negativity set, then we prove that $u$ has the asymptotic behavior

$$
\left.\left.u(x)=\alpha<x-x_{0}, \nu\right\rangle^{+}-\beta<x-x_{0}, \nu\right\rangle^{-}+o\left(\left|x-x_{0}\right|\right),
$$

where $\alpha, \beta>0$ and $\nu$ is the normal vector to the tangent ball at $x_{0}$ pointing inward $\{u>0\}$. The viscosity inequalities (1.6) then imply $\alpha=\beta$, that is $u$ is asymptotically a plane at $x_{0}$. This shows that any Lipschitz viscosity solution of (1.4) is also a viscosity solution to the following two phase free boundary problem

$$
\begin{cases}\mathcal{F}^{-}(u)=0 & \text { in }\{u>0\} \\ \mathcal{F}^{+}(u)=0 & \text { in }\{u<0\} \\ \frac{\partial u_{1}}{\partial \nu_{1}}=\frac{\partial u_{2}}{\partial \nu_{2}} & \text { on } \partial\{u>0\} \cap \Omega .\end{cases}
$$

We refer to [3] for the theory of viscosity solutions to free boundary problems. The regularity of the free boundary for problems of type (1.8) with same concave fully nonlinear operator in both the positivity and the negativity set of $u$ and with more general free boundary conditions, has been investigated, as already mentioned, in [20, 21]. More general operators have been considered in 1, 10,11.

Even though in (1.8) there are different operators on each side of the free boundary, we can still apply the results of [20,21] and prove that for any solution $u$ of (1.8) the following holds: if the free boundary is flat around a point $x_{0} \in \Gamma$, then in a neighborhood of $x_{0}$ it is a $C^{1, \alpha}$ surface. Going back to the original free boundary problem (1.4), this result implies that the set 
of regular points is an open subset of $\Gamma$ locally of class $C^{1, \alpha}$. In particular, $u$ has the asymptotic behavior (1.7) with $\alpha=\beta$ at any regular point.

To conclude, let us mention that we provide a simpler proof than in [19] of the existence of a Lipschitz solution of (1.4) that does not involve a segregation problem. Moreover as a byproduct of our results, we prove existence of a Lipschitz solution of (1.8). Existence of solutions to free boundary problems is in general a main issue. For (1.8), with $\mathcal{F}^{+}$replaced by $\mathcal{F}^{-}$it has been proven in [22]. We believe that our existence proofs could be generalized to a larger class of fully nonlinear operators.

1.1. Organization of the paper. The operators $\mathcal{F}^{-}$and $\mathcal{F}^{+}$are defined and their properties described in Section 2. Some examples are provided too. Our main results, Theorems 3.1 . 3.2 and 3.3, are contained in Section 3. In Section 4 we recall the monotonicity formula and some related results. Existence of a Lipschitz solution of the free boundary problem (1.8), i.e. Theorem 3.1, is proven in Section 5. In Section 6 we introduce the notion of regular points and we prove the non degeneracy of both $u_{1}$ and $u_{2}$ at regular points. Section 7 is devoted to the proof of Theorem 3.2. In Section 8 we prove that for the solution of (1.8) flat free boundaries are Lipschitz and, as a corollary, Theorem 3.3. Finally, some properties of the fundamental solution for the operator $\mathcal{F}^{-}$are proven in the Appendix.

\section{The operators $\mathcal{F}^{-}$And $\mathcal{F}^{+}$. Notation}

We will start by defining the two general fully nonlinear uniformly elliptic operators $\mathcal{F}^{-}$and $\mathcal{F}^{+}$. Let $\mathcal{S}_{n}$ be the set of symmetric $n \times n$ real matrices. Given $0<\lambda \leq 1<\Lambda$, let us denote by $\mathcal{A}_{\lambda, \Lambda}$ the set of matrices of $\mathcal{S}_{n}$ with eigenvalues in $[\lambda, \Lambda]$; i.e,

$$
\mathcal{A}_{\lambda, \Lambda}:=\left\{A \in \mathcal{S}_{n} \mid \lambda I_{n} \leq A \leq \Lambda I_{n}\right\},
$$

where $I_{n}$ is the identity matrix. Let $\mathcal{A}_{\lambda, \Lambda}^{1}$ and $\mathcal{A}_{\lambda, \Lambda}^{2}$ be two not empty subsets of $\mathcal{A}_{\lambda, \Lambda}$ with the property that

$$
\text { if } A \in \mathcal{A}_{\lambda, \Lambda}^{i}, i=1,2 \text {, and } O \in \mathcal{O}(n) \text {, then } O A O^{t} \in \mathcal{A}_{\lambda, \Lambda}^{i},
$$

where we denote by $\mathcal{O}(n)$ the set of $n \times n$ orthogonal matrices. Moreover, we assume that the identity matrix belongs to both sets,

$$
I_{n} \in \mathcal{A}_{\lambda, \Lambda}^{1} \cap \mathcal{A}_{\lambda, \Lambda}^{2}
$$

Let $\mathcal{F}^{+}$and $\mathcal{F}^{-}$be the following operators defined over matrices $M$ in $\mathcal{S}_{n}$,

$$
\mathcal{F}^{-}(M):=\inf _{A \in \mathcal{A}_{\lambda, \Lambda}^{1}} \operatorname{Tr}(A M)
$$

and

$$
\mathcal{F}^{+}(M):=\sup _{A \in \mathcal{A}_{\lambda, \Lambda}^{2}} \operatorname{Tr}(A M) .
$$

We remark that when $\mathcal{A}_{\lambda, \Lambda}^{1}=\mathcal{A}_{\lambda, \Lambda}^{2}=\mathcal{A}_{\lambda, \Lambda}$, then $\mathcal{F}^{-}=\mathcal{M}^{-}$and $\mathcal{F}^{+}=\mathcal{M}^{+}$, where $\mathcal{M}^{-}$and $\mathcal{M}^{+}$are the Pucci's extremal operators defined, for $M \in \mathcal{S}_{n}$, as follows

$$
\mathcal{M}^{-}(M)=\inf _{A \in \mathcal{A}_{\lambda, \Lambda}} \operatorname{Tr}(A M)=\lambda \sum_{e_{i}>0} e_{i}+\Lambda \sum_{e_{i}<0} e_{i}
$$

and

$$
\mathcal{M}^{+}(M)=\sup _{A \in \mathcal{A}_{\lambda, \Lambda}} \operatorname{Tr}(A M)=\Lambda \sum_{e_{i}>0} e_{i}+\lambda \sum_{e_{i}<0} e_{i}
$$


where $e_{i}, i=1, \ldots, n$ are the eigenvalues of the matrix $M$.

Proposition 2.1. $\mathcal{F}^{-}$and $\mathcal{F}^{+}$satisfy, for $M, N \in \mathcal{S}_{n}$

(a) $\mathcal{F}^{ \pm}(t M)=t \mathcal{F}^{ \pm}(M)$ for any $t \geq 0$;

(b) $\mathcal{F}^{+}(M+N) \leq \mathcal{F}^{+}(M)+\mathcal{F}^{+}(N)$ and hence $\mathcal{F}^{+}$is convex;

(c) $\mathcal{F}^{-}(M+N) \geq \mathcal{F}^{-}(M)+\mathcal{F}^{-}(N)$ and hence $\mathcal{F}^{-}$is concave;

(d) For any $M \in \mathcal{S}_{n}$,

$$
\mathcal{M}^{-}(M) \leq \mathcal{F}^{-}(M) \leq \operatorname{tr}(M) \leq \mathcal{F}^{+}(M) \leq \mathcal{M}^{+}(M)
$$

(e) (Uniformly Ellipticity) $\mathcal{M}^{-}(N) \leq \mathcal{F}^{ \pm}(M+N)-\mathcal{F}^{ \pm}(M) \leq \mathcal{M}^{+}(N)$;

Proof. Properties (a)-(c) are clear from the definitions (2.3) and (2.4) and the properties of the sup and inf functions.

Since $\mathcal{A}_{\lambda, \Lambda}^{1}, \mathcal{A}_{\lambda, \Lambda}^{2} \subset \mathcal{A}_{\lambda, \Lambda}$, we have that $\mathcal{M}^{-}(M) \leq \mathcal{F}^{-}(M)$ and $\mathcal{F}^{+}(M) \leq \mathcal{M}^{+}(M)$ for any $M \in \mathcal{S}_{n}$. Moreover, (2.2) implies that

$$
\mathcal{F}^{-}(M) \leq \operatorname{tr}(M) \leq \mathcal{F}^{+}(M) .
$$

This proves $(\mathrm{d})$.

By (b) and the last inequality in (d), we have that

$$
\mathcal{F}^{+}(M+N)-\mathcal{F}^{+}(M) \leq \mathcal{F}^{+}(N) \leq \mathcal{M}^{+}(N) .
$$

On the other hand, by the properties of the sup function,

$$
\mathcal{F}^{+}(M+N) \geq \mathcal{F}^{+}(M)+\inf _{A \in \mathcal{A}_{\lambda, \Lambda}^{2}} \operatorname{Tr}(A N) \geq \mathcal{F}^{+}(M)+\mathcal{M}^{-}(N) .
$$

This concludes the proof of (e) for $\mathcal{F}^{+}$. Similarly, one can prove (e) for $\mathcal{F}^{-}$.

Let $D$ be a domain of $\mathbb{R}^{n}$. With a slight abuse of notation, we define the differential operators, for $u \in C^{2}(D)$ and $x \in D$,

and

$$
\mathcal{F}^{-}(u)(x):=\mathcal{F}^{-}\left(D^{2} u(x)\right)
$$

$$
\mathcal{F}^{+}(u)(x):=\mathcal{F}^{+}\left(D^{2} u(x)\right),
$$

where $D^{2} u$ is the Hessian matrix of $u$. By Proposition 2.1, the differential operators $\mathcal{F}^{-}$and $\mathcal{F}^{+}$are 1 -homogeneous, uniformly elliptic, $\mathcal{F}^{-}$is concave and $\mathcal{F}^{+}$is convex. Moreover, by (d), for any $u \in C^{2}(D)$,

$$
\mathcal{M}^{-}(u) \leq \mathcal{F}^{-}(u) \leq \Delta u \leq \mathcal{F}^{+}(u) \leq \mathcal{M}^{+}(u),
$$

where again here we denote $\mathcal{M}^{-}(u)(x):=\mathcal{M}^{-}\left(D^{2} u(x)\right), \mathcal{M}^{+}(u)(x):=\mathcal{M}^{+}\left(D^{2} u(x)\right)$ and by $\Delta u$ the Laplacian of $u$. Furthermore, the operators $\mathcal{F}^{-}$and $\mathcal{F}^{+}$are invariant under rotations, as stated in the following proposition.

Proposition 2.2. Let $O$ be an orthogonal matrix. Let $u$ be $a C^{2}$-function and let $v(x)=u(O x)$. Then,

$$
\mathcal{F}^{ \pm}(v)(x)=\mathcal{F}^{ \pm}(u)(O x) .
$$

Proof. Since $D^{2} v(x)=O^{t} D^{2} u(O x) O$, we have that

$$
\begin{aligned}
\mathcal{F}^{-}(v)(x) & =\inf _{A \in \mathcal{A}_{\lambda, \Lambda}^{1}} \operatorname{tr}\left(A O^{t} D^{2} u(O x) O\right)=\inf _{A \in \mathcal{A}_{\lambda, \Lambda}^{1}} \operatorname{tr}\left(O A O^{t} D^{2} u(O x)\right) \\
& =\inf _{A \in \mathcal{A}_{\lambda, \Lambda}^{1}} \operatorname{tr}\left(A D^{2} u(O x)\right)=\mathcal{F}^{-}(u)(O x),
\end{aligned}
$$


where we have used that by (2.1),

$$
\mathcal{A}_{\lambda, \Lambda}^{1}=\left\{O A O^{t} \mid A \in \mathcal{A}_{\lambda, \Lambda}^{1}, O \in \mathcal{O}(n)\right\} .
$$

Similarly, $\mathcal{F}^{+}(v)(x)=\mathcal{F}^{+}(u)(O x)$.

Remark 2.3. By Proposition [2.1, Harnack inequality holds true for nonnegative viscosity solutions of $\mathcal{F}^{-}(u) \leq 0 \leq \mathcal{F}^{+}(u)$, see [Q, Theorem 4.3]. Observe also that $\mathcal{F}^{-}$and $\mathcal{F}^{+}$satisfy the comparison principle: if $D$ is a bounded domain and $u$ is a viscosity subsolution for $\mathcal{F}^{+}$in $D$, meaning $\mathcal{F}^{+}(u) \geq 0$ in the viscosity sense in $D, v$ is a viscosity supersolution for $\mathcal{F}^{+}$in $D$, meaning $\mathcal{F}^{+}(v) \leq 0$ in the viscosity sense $D$, and $u \leq v$ on $\partial D$ then $u \leq v$ in $\bar{D}$; the same result holds for $\mathcal{F}^{-}$, see [2, 9] for more details. In addition, since $\mathcal{F}^{-}$and $\mathcal{F}^{+}$are respectively concave and convex, interior $C^{2, \alpha}$-estimates for solutions of $\mathcal{F}^{ \pm}(u)=0$ hold true, see [2].

Remark 2.4. If $u$ is solution to (1.4), then

$$
\Gamma:=\partial\left\{u_{1}>0\right\} \cap \Omega=\partial\left\{u_{2}>0\right\} \cap \Omega .
$$

Indeed, if there was $x_{0} \in\left(\partial\left\{u_{1}>0\right\} \cap \Omega\right) \backslash \partial\left\{u_{2}>0\right\}$, then in a ball of radius $r$ around $x_{0}$ we would have

$$
\mathcal{F}^{-}\left(u_{1}\right)=\mathcal{F}^{-}(u) \leq 0, u_{1} \geq 0, u_{1} \not \equiv 0, u_{1}\left(x_{0}\right)=0 .
$$

This contradicts the strong maximum principle.

\subsection{Some examples.}

Example 2.5. As discussed in the Introduction, the free boundary problem (1.3), which is the limit problem of a population model studied in [19] that takes into account diffusion with preferential directions, is a particular case of problem (1.4). Indeed by choosing $\mathcal{A}_{\lambda, \Lambda}^{1}=\mathcal{A}_{\lambda, \Lambda}^{2}=$ $\mathcal{A}_{\lambda, \Lambda}$, we have that $\mathcal{F}^{-}=\mathcal{M}^{-}$and $\mathcal{F}^{+}=\mathcal{M}^{+}$.

Example 2.6. By choosing $\mathcal{A}_{\lambda, \Lambda}^{1}=\left\{I_{n}\right\}$ and $\mathcal{A}_{\lambda, \Lambda}^{2}=\mathcal{A}_{\lambda, \Lambda}$, problem (1.4) becomes

$$
\begin{cases}\Delta u=0 & \text { in }\{u>0\} \\ \mathcal{M}^{+}(u)=0 & \text { in }\{u<0\} \\ \Delta u \leq 0 & \text { in } \Omega \\ \mathcal{M}^{+}(u) \geq 0 & \text { in } \Omega \\ u=f & \text { on } \partial \Omega .\end{cases}
$$

Since the Bellman equations are very helpful to solve optimal stopping strategies see [16], this type of models can eventually be used to describe situations with multiple strategies.

Example 2.7. By the uniformly ellipticity, (e) in Proposition 2.1, the operators $\mathcal{F}^{+}$and $\mathcal{F}^{-}$ are Lipschitz continuous as functions in the space $\mathcal{S}(n)$. This regularity is optimal for the Pucci's operators $\mathcal{M}^{-}$and $\mathcal{M}^{+}$. Indeed, consider for example a family of matrices $\left\{M_{t} \mid t \in \mathbb{R}\right\}$ with eigenvalues $e_{1, t}=t$ and $e_{2, t}=e_{3, t}=\ldots=e_{n, t}=0$, then

$$
\mathcal{M}^{-}\left(M_{t}\right)= \begin{cases}\lambda t & \text { if } t \geq 0 \\ \Lambda t & \text { if } t<0\end{cases}
$$

which is a no more than Lipschitz function for $\lambda<\Lambda$. However there are operators in the class of extremal ones that we consider here which are more regular. Consider for example,

$$
\begin{aligned}
& \mathcal{F}^{-}(M)=\inf \left\{\operatorname{tr}(A M): A \in \mathcal{S}_{p}\right\} \\
& \mathcal{F}^{+}(M)=\sup \left\{\operatorname{tr}(A M): A \in \mathcal{S}_{p}\right\}
\end{aligned}
$$


where for $p>0$,

$$
\mathcal{A}_{\lambda, \Lambda}^{1}=\mathcal{A}_{\lambda, \Lambda}^{2}=\mathcal{S}_{p}:=\left\{A=\left(a_{i j}\right) \in \mathcal{S}_{n}:\left\|a_{i j}-\delta_{i j}\right\|_{l^{p}} \leq r_{0}<1\right\}
$$

for some $\lambda=\lambda\left(r_{0}\right)<1 \leq \Lambda$. Since, for example, for $p=2$ the balls in the $l^{2}$ norm are smooth, one can get a higher than Lipschitz regularity for $\mathcal{F}^{+}$and $\mathcal{F}^{-}$and thus, better than $C^{2, \alpha}$ estimates for the solutions $u$ of $\mathcal{F}^{ \pm}(u)=0$.

2.2. Notation. For a function $u, \nabla u$ and $D^{2} u$ denote respectively the gradient of $u$ and the Hessian matrix of $u$. The standard Euclidean inner produt is denoted by $\langle\cdot, \cdot\rangle$. We define $u^{+}:=\max (u, 0)$ and $u^{-}:=\max (-u, 0)$ which are the positive and negative part of $u$. In the rest of the paper, for the solution $u$ of (1.4), we will use the notation

$$
u_{1}:=u^{+} \quad \text { and } \quad u_{2}:=u^{-} \text {, }
$$

at our convenience. Notice that

$$
|u|(x)=\max \left(u_{1}(x), u_{2}(x)\right)=u_{1}(x)+u_{2}(x) .
$$

Furthermore, we denote by

$$
\Omega\left(u_{i}\right):=\left\{u_{i}>0\right\}
$$

$i=1,2$, the positivity set of $u_{i}$ and by

$$
\Gamma:=\partial\{u>0\} \cap \Omega,
$$

the free boundary set. If $u$ has an asymptotic development around $x_{0}$ along the direction $\nu_{1}$ given by,

we write that

$$
u(x)=\alpha<x-x_{0}, \nu_{1}>^{+}-\beta<x-x_{0}, \nu_{1}>^{-}+o\left(\left|x-x_{0}\right|\right)
$$

$$
\frac{\partial u_{1}}{\partial \nu_{1}}=\alpha \quad \text { and } \quad \frac{\partial u_{2}}{\partial \nu_{2}}=\beta
$$

where $\nu_{2}=-\nu_{1}$. We will consider the Euclidean norm for the distance, $d(x, y)=|x-y|$. Furthermore, we denote

$$
J_{r}\left(u_{i}, x_{0}\right):=\frac{1}{r^{2}} \int_{B_{r}\left(x_{0}\right)} \frac{\left|\nabla u_{i}\right|^{2}}{\left|x-x_{0}\right|^{n-2}} d x
$$

and

$$
J_{r}\left(u, x_{0}\right):=J_{r}\left(u_{1}, x_{0}\right) J_{r}\left(u_{2}, x_{0}\right) .
$$

When $x_{0}=0$ we simply write $J_{r}(u)$ instead of $J_{r}(u, 0)$.

\section{MAin RESUlts}

Theorem 3.1. Let $\Omega$ be a bounded smooth domain of $\mathbb{R}^{n}$ and $f$ be a Lipschitz continuous function on $\partial \Omega$ such that $f^{+} \not \equiv 0$ and $f^{-} \not \equiv 0$. Then there exists a viscosity solution $u$ of (1.4) such that $u_{1}=u^{+} \not \equiv 0$ and $u_{2}=u^{-} \not \equiv 0$. Moreover $u$ is Lipschitz continuous in $\bar{\Omega}$.

Theorem 3.2. Any Lipschitz solution $u$ of (1.4) such that $u_{1}=u^{+} \not \equiv 0$ and $u_{2}=u^{-} \not \equiv 0$, satisfies in the viscosity sense the free boundary condition

$$
\frac{\partial u_{1}}{\partial \nu_{1}}=\frac{\partial u_{2}}{\partial \nu_{2}} \quad \text { on } \Gamma=\partial\{u>0\} \cap \Omega,
$$

meaning that: if there exists a tangent ball $B$ at $x_{0} \in \Gamma$, such that either $B \subset \Omega\left(u_{1}\right)$ or $B \subset \Omega\left(u_{2}\right)$, then there exists $\alpha>0$ such that

$$
u(x)=\alpha<x-x_{0}, \nu_{1}>+o\left(\left|x-x_{0}\right|\right)
$$


where $\nu_{1}$ is the normal vector to $\partial B$ at $x_{0}$ pointing inward to $\Omega\left(u_{1}\right)$ (and $\left.\nu_{2}=-\nu_{1}\right)$. In particular, $u$ is a viscosity solution to the free boundary problem (1.8).

Theorem 3.3. Let $u$ be any Lipschitz solution of (1.4) and let $\mathcal{R}$ be the set of regular points of $u$, according to Definition 6.1. Then $\mathcal{R}$ is an open subset of $\Gamma$ and locally a surface of class $C^{1, \alpha}$, with $0<\alpha \leq 1$. In particular, $u$ has the asymptotic behavior (3.1) at any $x_{0} \in \mathcal{R}$.

The proofs of Theorems $3.1,3.2$ and 3.3 are given respectively in Sections 5 , 7 and 8 .

\section{Backround: The monotonicity Formula}

In this section we recall the Alt-Caffarelli-Friedman monotonicity formula and some related results that we will used later on in the paper. A proof can be found in [3, 18. We have:

Theorem 4.1 (Monotonicity formula). Let $u_{1}, u_{2} \in C\left(B_{1}(0)\right)$ be nonnegative subharmonic functions in $B_{1}(0)$. Assume $u_{1} u_{2}=0$ and $u_{1}(0) u_{2}(0)=0$. Let $u=u_{1}-u_{2}$ and

$$
J_{r}(u)=\frac{1}{r^{4}} \int_{B_{r}(0)} \frac{\left|\nabla u_{1}\right|^{2}}{|x|^{n-2}} d x \int_{B_{r}(0)} \frac{\left|\nabla u_{2}\right|^{2}}{|x|^{n-2}} d x, \quad 0<r<1 .
$$

Then $J_{r}(u)$ is finite and is a non-decreasing function of $r$. Moreover,

$$
J_{r}(u) \leq c(n)\left\|u_{1}\right\|_{L^{2}\left(B_{1}\right)}^{2}\left\|u_{2}\right\|_{L^{2}\left(B_{1}\right)}^{2}, \quad 0<r \leq \frac{1}{2} .
$$

Theorem 4.1 can be applied to $u=u_{1}-u_{2}$ solution of (1.4). Indeed, by (2.5) we have $\Delta u_{1} \geq \mathcal{F}^{-}\left(u_{1}\right)=0$ in $\Omega\left(u_{1}\right)$ and $\Delta u_{2} \geq-\mathcal{F}^{+}\left(-u_{2}\right)=0$ in $\Omega\left(u_{2}\right)$. Therefore, both $u_{1}$ and $u_{2}$ are subharmonic functions in the viscosity sense, and thus is in the distributional sense, in the whole $\Omega$,

$$
\Delta u_{1} \geq 0 \quad \text { in } \Omega
$$

$$
\Delta u_{2} \geq 0 \quad \text { in } \Omega \text {. }
$$

Remark 4.2. Since $J_{r}(u)$ is a monotone nonnegative function, there exists

$$
J_{0}(u):=\lim _{r \rightarrow 0^{+}} J_{r}(u) .
$$

The following theorem gives information on the case $J_{r}(u)$ constant. A proof of it can be found in [18].

Theorem 4.3. Let $u_{1}, u_{2} \in C\left(B_{1}(0)\right)$ be nonnegative subharmonic functions in $B_{1}(0)$. Assume $u_{1} u_{2}=0$ and $u_{1}(0) u_{2}(0)=0$ and let $u=u_{1}-u_{2}$. If

$$
J_{r_{1}}(u)=J_{r_{2}}(u)=: k
$$

for some $0<r_{1}<r_{2}<1$, then, either one or the other of the following holds:

(i) $u_{1} \equiv 0$ in $B_{r_{2}}(0)$ or $u_{2} \equiv 0$ in $B_{r_{2}}(0)$;

(ii) there exist a unit vector $\nu$, positive constants $\alpha, \beta$ and a universal positive constant $c_{n}$, such that

and for any $x \in B_{r_{2}}(0)$,

$$
k=c_{n} \alpha^{2} \beta^{2}
$$

$$
\left.u(x)=\alpha<x, \nu>^{+}-\beta<x, \nu\right\rangle^{-} .
$$




\section{Proof of Theorem 3.1}

We consider the Heaviside function $H: \mathbb{R} \rightarrow\{0,1\}$,

$$
H(x)=\left\{\begin{array}{l}
1 \text { when } x \geq 0 \\
0 \text { when } x<0,
\end{array}\right.
$$

and we let $H_{\epsilon}$ denote a smooth approximation of $H$, satisfying $H_{\epsilon}^{\prime} \geq 0$. Consider the fully nonlinear uniformly elliptic operator $G$, defined by

$$
G(u):=H(u) \mathcal{F}^{-}(u)+(1-H(u)) \mathcal{F}^{+}(u)
$$

and its $\epsilon$-approximation $G_{\epsilon}$, defined by

$$
G_{\epsilon}(u):=H_{\epsilon}(u) \mathcal{F}^{-}(u)+\left(1-H_{\epsilon}(u)\right) \mathcal{F}^{+}(u) .
$$

To prove existence of a Lipschitz solution of (1.4), we prove that for any $\epsilon>0$, there exists $u^{\epsilon}$ viscosity solution of the problem

$$
\begin{cases}G_{\epsilon}\left(u^{\epsilon}\right)=0 & \text { in } \Omega \\ u^{\epsilon}=f & \text { on } \partial \Omega,\end{cases}
$$

and that the functions $u^{\epsilon}$ 's are Lipschitz continuous uniformly in $\epsilon$. Existence of a solution of (1.4) will then follow by using the Ascoli-Arzelà Theorem and the stability of the viscosity solutions in the sets $\{u>0\}$ and $\{u<0\}$.

Remark 5.1. By Proposition 2.1, in the viscosity sense

$$
\mathcal{M}^{-}\left(u^{\epsilon}\right) \leq \mathcal{F}^{-}\left(u^{\epsilon}\right) \leq G_{\epsilon}\left(u^{\epsilon}\right) \leq \mathcal{F}^{+}\left(u^{\epsilon}\right) \leq \mathcal{M}^{+}\left(u^{\epsilon}\right)
$$

We start by proving that any viscosity solution of (5.3) is Lipschitz continuous with Lipschitz norm independent of $\epsilon$.

Theorem 5.2. Let $\epsilon>0, \Omega$ be a bounded smooth domain and $G_{\epsilon}$ the operator defined in (5.2). Let $f \in C^{0,1}(\partial \Omega)$ satisfy

$$
\|f\|_{C^{0,1}(\partial \Omega)} \leq K_{0} .
$$

Then, any continuous viscosity solution $u^{\epsilon}$ of problem (5.3) is Lipschitz continuous in $\bar{\Omega}$ and

$$
\left\|u^{\epsilon}\right\|_{C^{0,1}(\bar{\Omega})} \leq C
$$

where $C=C\left(n, \Omega, \lambda, \Lambda, K_{0}\right)$.

Proof. Before giving the precise proof, we will give an heuristic argument, just to give an idea of the main technic. Assume that $u^{\epsilon}$ has a further regularity, for instance $u^{\epsilon} \in C^{3}(\Omega)$. Since $\mathcal{F}^{-}(M)$ and $\mathcal{F}^{+}(M)$ are Lipschitz continuous with respect to $M \in \mathcal{A}_{n}$, we have that $\mathcal{F}^{-}\left(u^{\epsilon}\right)$ and $\mathcal{F}^{-}\left(u^{\epsilon}\right)$ are Lipschitz continuous with respect to $x$, therefore we can differentiate a.e. in $\Omega$ both sides of the equation

$$
G_{\epsilon}\left(u^{\epsilon}\right)=0,
$$

in any direction $\sigma \in \partial B_{1}(0)$. Indeed, if we denote

$$
\mathcal{F}_{i j}^{ \pm}(M):=\frac{\partial \mathcal{F}^{ \pm}}{\partial m_{i j}}(M),
$$

where $M=\left(m_{i j}\right)$, we obtain

$$
\begin{aligned}
0=\partial_{\sigma} G_{\epsilon}\left(u^{\epsilon}\right) & =H_{\epsilon}\left(u^{\epsilon}\right) \mathcal{F}^{-}{ }_{i j}\left(u^{\epsilon}\right) \partial_{i j}\left(\partial_{\sigma} u^{\epsilon}\right)+\left(1-H_{\epsilon}\left(u^{\epsilon}\right)\right) \mathcal{F}^{+}{ }_{i j}\left(u^{\epsilon}\right) \partial_{i j}\left(\partial_{\sigma} u^{\epsilon}\right) \\
& +H_{\epsilon}^{\prime}\left(u^{\epsilon}\right)\left(\mathcal{F}^{-}\left(u^{\epsilon}\right)-\mathcal{F}^{+}\left(u^{\epsilon}\right)\right) \partial_{\sigma} u^{\epsilon}
\end{aligned}
$$


Then, if $L$ denotes the linear operator, with coefficients that depend on $u^{\epsilon}$,

$$
L(\cdot):=\left(H_{\epsilon}\left(u^{\epsilon}\right) \mathcal{F}^{-}{ }_{i j}\left(u^{\epsilon}\right)+\left(1-H_{\epsilon}\left(u^{\epsilon}\right)\right) \mathcal{F}^{+}{ }_{i j}\left(u^{\epsilon}\right)\right) \partial_{i j}(\cdot),
$$

we can see that $\partial_{\sigma} u^{\epsilon}$ is a solution of

$$
L\left(\partial_{\sigma} u^{\epsilon}\right)+\underbrace{H_{\epsilon}^{\prime}\left(u^{\epsilon}\right)\left(\mathcal{F}^{-}\left(u^{\epsilon}\right)-\mathcal{F}^{+}\left(u^{\epsilon}\right)\right)}_{\leq 0} \partial_{\sigma} u^{\epsilon}=0 .
$$

Since $H_{\epsilon}^{\prime}\left(u^{\epsilon}\right)\left(\mathcal{F}^{-}\left(u^{\epsilon}\right)-\mathcal{F}^{+}\left(u^{\epsilon}\right)\right) \leq 0$ by the maximum principle,

$$
\sup _{\Omega} \partial_{\sigma} u^{\epsilon} \leq \sup _{\partial \Omega} \partial_{\sigma} u^{\epsilon}
$$

Now, if $\sigma$ is a tangential direction to $\partial \Omega$, then

$$
\sup _{\partial \Omega} \partial_{\sigma} u^{\epsilon}=\sup _{\partial \Omega} \partial_{\sigma} f \leq\|\nabla f\|_{L^{\infty}(\partial \Omega)} .
$$

If $\sigma$ is a normal vector, then a barrier argument (as the one in Claim 1 below) shows that

$$
\sup _{\partial \Omega} \partial_{\sigma} u^{\epsilon} \leq C
$$

Thus, $\partial_{\sigma} u^{\epsilon}$ is bounded in $\Omega$ and the arbitrarily of $\sigma$ implies the result. To overcome the lack of regularity, we will use standard techniques from the theory of viscosity solutions. In particular, we will discretize and prove that the incremental quotient is bounded, meaning that there exists a constant $C_{0}$ independent of $\epsilon$ such that, for $\sigma \in \partial B_{1}(0)$ and $h>0$ :

$$
\sup _{x, x+h \sigma \in \Omega} \frac{u^{\epsilon}(x+\sigma h)-u^{\epsilon}(x)}{h} \leq C_{0} .
$$

Then the result holds true. To prove (5.4) we first prove the following claim:

Claim 1 : There exists $C_{0}>0$ independent of $\epsilon$ such that for any $x \in \bar{\Omega}$ and any $y \in \partial \Omega$,

$$
\left|u^{\epsilon}(x)-u^{\epsilon}(y)\right| \leq C_{0}|x-y| \text {. }
$$

Proof of Claim 1: Consider the function $\psi$ solution to

$$
\begin{cases}\mathcal{M}^{-}(\psi)=0 & \text { in } \Omega \\ \psi=f & \text { on } \partial \Omega .\end{cases}
$$

Then, $\psi \in C^{2, \alpha}(\Omega)$ and $\psi \in C^{0,1}(\bar{\Omega})$, see [2,12]. Remark 5.1] and comparison principle implies $u^{\epsilon} \geq \psi$ in $\bar{\Omega}$. In particular, if $y \in \partial \Omega$ and $x \in \Omega$, we have

$$
u^{\epsilon}(x)-u^{\epsilon}(y) \geq \psi(x)-\psi(y) \geq-C_{0}|x-y|,
$$

for some $C_{0}>0$ independent of $\epsilon$. Similarly, the inequality

$$
u^{\epsilon}(x)-u^{\epsilon}(y) \leq C_{0}|x-y|
$$

follows by comparing $u^{\epsilon}$ with the solution $\varphi$ of

$$
\begin{cases}\mathcal{M}^{+}(\varphi)=0 & \text { in } \Omega \\ \varphi=f & \text { on } \partial \Omega .\end{cases}
$$

Claim 1 follows from estimates (5.5) and (5.6). 
Next, to prove (5.4), assume by contradiction that, for some $\delta>0$,

$$
\sup _{x, x+h \sigma \in \Omega} \frac{u^{\epsilon}(x+h \sigma)-u^{\epsilon}(x)}{h} \geq C_{0}+\delta,
$$

where $C_{0}>0$ is given in Claim 1. Then, for $\kappa>0$, we have that

$$
\sup _{x, x+h \sigma \in \Omega} \frac{u^{\epsilon}(x+h \sigma)+\kappa|x|^{2}-u^{\epsilon}(x)}{h} \geq C_{0}+\delta .
$$

In what follows we will make explicit the dependence of the operator $G_{\epsilon}(u)$ in $u$ and $M \in \mathcal{S}_{n}$ by using the notation $G_{\epsilon}(u, M)$. Denote $w_{h}^{\epsilon}(x):=u^{\epsilon}(x+h \sigma)+\kappa|x|^{2}$. Note that, by the uniformly ellipticity and the fact that $u^{\epsilon}$ is solution of (5.3), $w_{h}^{\epsilon}$ is a strict subsolution of $G\left(w_{h}^{\epsilon}-\kappa|x|^{2}, D^{2} w_{h}^{\epsilon}\right)=0$, in $\Omega-h \sigma:=\{x-h \sigma \mid x \in \Omega\}$ as it satisfies in the viscosity sense

$$
\begin{aligned}
0 & =G\left(w_{h}^{\epsilon}-\kappa|x|^{2}, D^{2} w_{h}^{\epsilon}-2 \kappa I_{n}\right) \leq G\left(w_{h}^{\epsilon}-\kappa|x|^{2}, D^{2} w_{h}^{\epsilon}\right)-\mathcal{M}^{-}\left(2 \kappa I_{n}\right) \\
& =G\left(w_{h}^{\epsilon}-\kappa|x|^{2}, D^{2} w_{h}^{\epsilon}\right)-2 \kappa \lambda n,
\end{aligned}
$$

from which

$$
G_{\epsilon}\left(w_{h}^{\epsilon}-\kappa|x|^{2}, D^{2} w_{h}^{\epsilon}\right) \geq 2 \kappa \lambda n \quad \text { in } \Omega-h \sigma .
$$

In order to infer a differential inequality satisfied by $w_{h}^{\epsilon}(x)-u^{\epsilon}(x)$ in the viscosity sense, consider for any fixed $\tau_{0}>0$ and $0<\tau<\tau_{0}$, the upper $\tau$-envelope of $w_{h}^{\epsilon}$ and the lower $\tau$-envelope of $u^{\epsilon}$ defined respectively by

$$
\begin{array}{cl}
w^{\tau}(x):=\sup _{y \in \Omega_{\tau_{0}}-h \sigma}\left\{w_{h}^{\epsilon}(y)+\tau-\frac{1}{\tau}|y-x|^{2}\right\}, & x \in \Omega_{\tau_{0}}-h \sigma \\
u_{\tau}(x):=\inf _{y \in \Omega_{\tau_{0}}}\left\{u^{\epsilon}(y)-\tau+\frac{1}{\tau}|y-x|^{2}\right\}, & x \in \Omega_{\tau_{0}},
\end{array}
$$

where $\Omega_{\tau_{0}}:=\left\{x \in \Omega \mid d(x, \partial \Omega)>\tau_{0}\right\}$ and, for simplicity of notation, we have dropped the dependence on $h$ and $\epsilon$.

Claim 2 : The upper and lower $\tau$-envelopes have the following properties:

a) $w^{\tau} \in C\left(\Omega_{\tau_{0}}-h \sigma\right), u_{\tau} \in C\left(\Omega_{\tau_{0}}\right), w^{\tau} \geq w_{h}^{\epsilon}+\tau, u_{\tau} \leq u^{\epsilon}-\tau, w^{\tau} \rightarrow w_{h}^{\epsilon}$ and $u_{\tau} \rightarrow u^{\epsilon}$ as $\tau \rightarrow 0$ uniformly in $\Omega_{\tau_{0}}-h \sigma$ and in $\Omega_{\tau_{0}}$ respectively.

b) For any $x \in \Omega_{\tau_{0}} \cap\left(\Omega_{\tau_{0}}-h \sigma\right)$ there exists a concave (resp., convex) paraboloid of opening $2 / \tau$ that touches $w^{\tau}$ (resp., $u_{\tau}$ ) by below (resp., above) at $x$. In particular, $w^{\tau}$ and $u_{\tau}$ are punctually second order differentiable almost everywhere in $\Omega_{\tau_{0}} \cap\left(\Omega_{\tau_{0}}-h \sigma\right)$, meaning that, for a.e. $x_{0} \in \Omega_{\tau_{0}} \cap\left(\Omega_{\tau_{0}}-h \sigma\right)$ there exist paraboloids $P_{w}$ and $P_{u}$, such that, $w^{\tau}(x)=P_{w}(x)+o\left(\left|x-x_{0}\right|^{2}\right)$ and $u_{\tau}(x)=P_{u}(x)+o\left(\left|x-x_{0}\right|^{2}\right)$ as $x \rightarrow x_{0}$.

c) If $x^{\tau} \in \Omega_{\tau_{0}}-h \sigma$ is such that $w^{\tau}(x)=w_{h}^{\epsilon}\left(x^{\tau}\right)+\tau-\frac{1}{\tau}\left|x-x^{\tau}\right|^{2}$, then

$$
\frac{1}{\tau}\left|x-x^{\tau}\right|^{2} \leq w_{h}^{\epsilon}\left(x^{\tau}\right)-w_{h}^{\epsilon}(x) .
$$

If $x_{\tau} \in \Omega_{\tau_{0}}$ is such that $u_{\tau}(x)=u^{\epsilon}\left(x_{\tau}\right)-\tau+\frac{1}{\tau}\left|x-x_{\tau}\right|^{2}$, then

$$
\frac{1}{\tau}\left|x-x_{\tau}\right|^{2} \leq u^{\epsilon}(x)-u^{\epsilon}\left(x_{\tau}\right) \text {. }
$$


d) There exists $\tau_{1}>0$ such that for any $\tau<\tau_{1}, w^{\tau}$ is a viscosity (and therefore a.e.) subsolution to

$$
G_{\epsilon}\left(w^{\tau}(x)-\tau+\frac{1}{\tau}\left|x-x^{\tau}\right|^{2}-\kappa\left|x^{\tau}\right|^{2}, D^{2} w^{\tau}(x)\right)=2 \kappa \lambda n, \quad x \in \Omega_{2 \tau_{0}}-h \sigma
$$

and $u_{\tau}$ is a viscosity (and therefore a.e.) supersolution to

$$
G_{\epsilon}\left(u_{\tau}(x)+\tau-\frac{1}{\tau}\left|x-x_{\tau}\right|^{2}, D^{2} u_{\tau}(x)\right)=0, \quad x \in \Omega_{2 \tau_{0}}
$$

Proof of Claim 2: For the proofs of (a)-(c) see Theorem 5.1 and Lemma 5.2 in [2]. Note that by $(c)$,

$$
\frac{1}{\tau}\left|x-x^{\tau}\right|^{2} \leq w_{h}^{\epsilon}\left(x_{\tau}\right)-w_{h}^{\epsilon}(x) \leq C .
$$

Since $w_{h}^{\epsilon}$ is continuous this implies that

$$
\frac{1}{\tau}\left|x-x^{\tau}\right|^{2} \rightarrow 0 \quad \text { as } \tau \rightarrow 0 .
$$

Similarly,

$$
\frac{1}{\tau}\left|x-x_{\tau}\right|^{2} \rightarrow 0 \quad \text { as } \tau \rightarrow 0 .
$$

To prove (d), let $x_{0} \in \Omega_{2 \tau_{0}}-h \sigma$ and let $P(x)$ be a paraboloid that touches by above $w^{\tau}$ at $x_{0}$. Consider the paraboloid

$$
Q(x)=P\left(x+x_{0}-x_{0}^{\tau}\right)+\frac{1}{\tau}\left|x_{0}-x_{0}^{\tau}\right|^{2}-\tau .
$$

By (5.9), we can pick $\tau_{1}>0$ independent of $x_{0}$ such that $x_{0}^{\tau} \in \Omega_{\tau_{0}}-h \sigma$ for any $\tau<\tau_{1}$. Take any $x$ sufficiently close to $x_{0}^{\tau}$, so that $x+x_{0}-x_{0}^{\tau} \in \Omega_{\tau_{0}}-h \sigma$, then, by definition of $w^{\tau}$,

$$
w_{h}^{\epsilon}(x) \leq w^{\tau}\left(x+x_{0}-x_{0}^{\tau}\right)+\frac{1}{\tau}\left|x_{0}-x_{0}^{\tau}\right|^{2}-\tau \leq Q(x)
$$

Moreover, $w_{h}^{\epsilon}\left(x_{0}^{\tau}\right)=Q\left(x_{0}^{\tau}\right)$, since $w^{\tau}\left(x_{0}\right)=P\left(x_{0}\right)$. Hence $Q$ touches $w_{h}^{\epsilon}$ by above at $x_{0}^{\tau}$ and by (5.8),

$$
2 \kappa \lambda n \leq G_{\epsilon}\left(w_{h}^{\epsilon}\left(x_{0}^{\tau}\right)-\kappa\left|x_{0}^{\tau}\right|^{2}, D^{2} Q\left(x_{0}^{\tau}\right)\right)=G_{\epsilon}\left(w^{\tau}\left(x_{0}\right)-\tau+\frac{1}{\tau}\left|x_{0}-x_{0}^{\tau}\right|^{2}-\kappa\left|x_{0}^{\tau}\right|^{2}, D^{2} P\left(x_{0}\right)\right) .
$$

Similarly one can prove the second viscosity inequality in (d). This concludes the proof of Claim 2 .

Let us continue the proof of the theorem. We have assumed that (5.7) is true. If the supremum in (5.7) is attained at $\bar{x}$, then both $\bar{x}$ and $\bar{x}+\sigma h$ have to be in the interior of $\Omega$, for otherwise we would have $u^{\epsilon}(\bar{x}+h \sigma)-u^{\epsilon}(\bar{x}) \geq C_{0} h+\delta h-\kappa|\bar{x}|^{2} \geq C_{0} h+\delta h-\kappa C(\Omega)$ which contradicts Claim 1 for $\kappa<\delta h / C(\Omega)$. Thus, there exists $\tau_{0}>0$ such that

$$
\sup _{x+h \sigma, x \in \Omega} \frac{w_{h}^{\epsilon}(x)-u^{\epsilon}(x)}{h}=\sup _{x+h \sigma, x \in \Omega_{3 \tau_{0}}} \frac{w_{h}^{\epsilon}(x)-u^{\epsilon}(x)}{h} \geq C_{0}+\delta .
$$

For $\tau$ small enough, by (a) of Claim 2, there exists $x_{0} \in \Omega_{\frac{5 \tau_{0}}{2}} \cap\left(\Omega_{\frac{5 \tau_{0}}{2}}-h \sigma\right)$ such that

$$
\sup _{x+h \sigma, x \in \Omega_{\tau_{0}}} \frac{w^{\tau}(x)-u_{\tau}(x)}{h}=\frac{w^{\tau}\left(x_{0}\right)-u_{\tau}\left(x_{0}\right)}{h}=M \geq C_{0} .
$$


Take $s>0$ and $r<\tau_{0} / 2$ small enough so that $B_{r}\left(x_{0}\right) \subset \Omega_{2 \tau_{0}} \cap\left(\Omega_{2 \tau_{0}}-h \sigma\right)$ and define

$$
v(x):=u_{\tau}(x)-w^{\tau}(x)+M h+s\left|x-x_{0}\right|^{2}-s r^{2} .
$$

Then $v$ has a strict minimum at $x_{0}$, moreover

$$
v(x) \geq 0 \quad \text { on } \partial B_{r}\left(x_{0}\right) \text { and } v\left(x_{0}\right)=-s r^{2}<0 .
$$

Let us denote by $\Gamma_{v}$ the convex envelope of $-v^{-}$in $B_{2 r}\left(x_{0}\right)$, where we have extended $v \equiv 0$ outside $B_{r}\left(x_{0}\right)$. Here we use standard techniques from the theory of viscosity solutions, see [2]. Since we do not know if $w^{\tau}$ and $u_{\tau}$ are twice differentiable at $x_{0}$, we introduce the convex envelope in order to find a point $x_{1}$ of twice differentiability for both $w^{\tau}$ and $u_{\tau}$ such that $w^{\tau}\left(x_{1}\right)>u_{\tau}\left(x_{1}\right)$ and $D^{2} w^{\tau}\left(x_{1}\right) \leq D^{2} u_{\tau}\left(x_{1}\right)+$ small corrections. We have that $\Gamma_{v} \leq 0$ in $B_{2 r}\left(x_{0}\right)$. By (b) of Claim 2, for any $x \in \overline{B_{r}\left(x_{0}\right)} \cap\left\{v=\Gamma_{v}\right\}$ there exists a convex paraboloid with opening independent of $x$ that touches $\Gamma_{v}$ by above. By Lemma 3.5 in [2], $\Gamma_{v} \in C^{1,1}\left(B_{r}\left(x_{0}\right)\right)$ and

$$
\int_{B_{r}\left(x_{0}\right) \cap\left\{v=\Gamma_{v}\right\}} \operatorname{det} D^{2} \Gamma_{v} d x>0 .
$$

In particular $\left|B_{r}\left(x_{0}\right) \cap\left\{v=\Gamma_{v}\right\}\right|>0$. Since $w^{\tau}$ and $u_{\tau}$ are second order differentiable almost everywhere in $B_{r}\left(x_{0}\right)$, there exists $x_{1} \in B_{r}\left(x_{0}\right) \cap\left\{v=\Gamma_{v}\right\}$ such that $w^{\tau}$ and $u_{\tau}$ are second order differentiable at $x_{1}$ and by (d) of Claim 2 ,

$$
G_{\epsilon}\left(w^{\tau}\left(x_{1}\right)-\tau+\frac{1}{\tau}\left|x_{1}-x_{1}^{\tau}\right|^{2}-\kappa\left|x_{1}^{\tau}\right|^{2}, D^{2} w^{\tau}\left(x_{1}\right)\right) \geq 2 \kappa \lambda n
$$

and

$$
G_{\epsilon}\left(u_{\tau}\left(x_{1}\right)+\tau-\frac{1}{\tau}\left|x_{1}-\left(x_{1}\right)_{\tau}\right|^{2}, D^{2} u_{\tau}\left(x_{1}\right)\right) \leq 0 .
$$

Since $\Gamma_{v}$ is convex, $\Gamma_{v} \leq v$ and $\Gamma_{v}\left(x_{1}\right)=v\left(x_{1}\right)$, we have that $D^{2} v\left(x_{1}\right) \geq 0$, i.e.,

$$
D^{2} w^{\tau}\left(x_{1}\right) \leq D^{2} u_{\tau}\left(x_{1}\right)+2 s I_{n} .
$$

Moreover, since $\Gamma_{v}$ is negative in $B_{r}\left(x_{0}\right)$, we have that

$$
w^{\tau}\left(x_{1}\right)>u_{\tau}\left(x_{1}\right)+s\left|x_{1}-x_{0}\right|^{2}-s r^{2}+M h .
$$

In particular, for $s$ and $r$ small enough

$$
w^{\tau}\left(x_{1}\right)>u_{\tau}\left(x_{1}\right)+\frac{M h}{2} .
$$

Let us denote $\varphi^{\tau}\left(x_{1}\right):=-\tau+\frac{1}{\tau}\left|x_{1}-x_{1}^{\tau}\right|^{2}$ and $\varphi_{\tau}\left(x_{1}\right):=\tau-\frac{1}{\tau}\left|x_{1}-\left(x_{1}\right)_{\tau}\right|^{2}$. Then, by subtracting the inequalities (5.13) and (5.14), we get

$$
\begin{aligned}
2 \kappa \lambda n & \leq G_{\epsilon}\left(w^{\tau}\left(x_{1}\right)+\varphi^{\tau}\left(x_{1}\right)-\kappa\left|x_{1}^{\tau}\right|^{2}, D^{2} w^{\tau}\left(x_{1}\right)\right)-G_{\epsilon}\left(u_{\tau}\left(x_{1}\right)+\varphi_{\tau}\left(x_{1}\right), D^{2} u_{\tau}\left(x_{1}\right)\right) \\
& =H_{\epsilon}\left(w^{\tau}\left(x_{1}\right)+\varphi^{\tau}\left(x_{1}\right)-\kappa\left|x_{1}^{\tau}\right|^{2}\right) \mathcal{F}^{-}\left(w^{\tau}\right)\left(x_{1}\right) \\
& +\left(1-H_{\epsilon}\left(w^{\tau}\left(x_{1}\right)+\varphi^{\tau}\left(x_{1}\right)-\kappa\left|x_{1}^{\tau}\right|^{2}\right)\right) \mathcal{F}^{+}\left(w^{\tau}\right)\left(x_{1}\right) \\
& -H_{\epsilon}\left(u_{\tau}\left(x_{1}\right)+\varphi_{\tau}\left(x_{1}\right)\right) \mathcal{F}^{-}\left(u_{\tau}\right)\left(x_{1}\right)-\left(1-H_{\epsilon}\left(u_{\tau}\left(x_{1}\right)+\varphi_{\tau}\left(x_{1}\right)\right)\right) \mathcal{F}^{+}\left(u_{\tau}\right)\left(x_{1}\right) .
\end{aligned}
$$

Adding and subtracting

and

$$
H_{\epsilon}\left(u_{\tau}\left(x_{1}\right)+\varphi_{\tau}\left(x_{1}\right)\right) \mathcal{F}^{-}\left(w^{\tau}\right)\left(x_{1}\right)
$$

$$
\left[1-H_{\epsilon}\left(u_{\tau}\left(x_{1}\right)+\varphi_{\tau}\left(x_{1}\right)\right)\right] \mathcal{F}^{+}\left(w^{\tau}\right)\left(x_{1}\right),
$$

in the right hand-side of the inequality above, we obtain 


$$
\begin{aligned}
2 \kappa \lambda n & \leq\left[H_{\epsilon}\left(w^{\tau}\left(x_{1}\right)+\varphi^{\tau}\left(x_{1}\right)-\kappa\left|x_{1}^{\tau}\right|^{2}\right)-H_{\epsilon}\left(u_{\tau}\left(x_{1}\right)+\varphi_{\tau}\left(x_{1}\right)\right)\right] \mathcal{F}^{-}\left(w^{\tau}\right)\left(x_{1}\right) \\
& +H_{\epsilon}\left(u_{\tau}\left(x_{1}\right)+\varphi_{\tau}\left(x_{1}\right)\right) \mathcal{F}^{-}\left(w^{\tau}\right)\left(x_{1}\right) \\
& -\left[H_{\epsilon}\left(w^{\tau}\left(x_{1}\right)+\varphi^{\tau}\left(x_{1}\right)-\kappa\left|x_{1}^{\tau}\right|^{2}\right)-H_{\epsilon}\left(u_{\tau}\left(x_{1}\right)+\varphi_{\tau}\left(x_{1}\right)\right)\right] \mathcal{F}^{+}\left(w^{\tau}\right)\left(x_{1}\right) \\
& +\left[1-H_{\epsilon}\left(u_{\tau}\left(x_{1}\right)+\varphi_{\tau}\left(x_{1}\right)\right)\right] \mathcal{F}^{+}\left(w^{\tau}\right)\left(x_{1}\right) \\
& -H_{\epsilon}\left(u_{\tau}\left(x_{1}\right)+\varphi_{\tau}\left(x_{1}\right)\right) \mathcal{F}^{-}\left(u_{\tau}\right)\left(x_{1}\right)-\left(1-H_{\epsilon}\left(u_{\tau}\left(x_{1}\right)+\varphi_{\tau}\left(x_{1}\right)\right)\right) \mathcal{F}^{+}\left(u_{\tau}\right)\left(x_{1}\right) \\
& =\left[H_{\epsilon}\left(w^{\tau}\left(x_{1}\right)+\varphi^{\tau}\left(x_{1}\right)-\kappa\left|x_{1}^{\tau}\right|^{2}\right)-H_{\epsilon}\left(u_{\tau}\left(x_{1}\right)+\varphi_{\tau}\left(x_{1}\right)\right)\right] \mathcal{F}^{-}\left(w^{\tau}\right)\left(x_{1}\right) \\
& -\left[H_{\epsilon}\left(w^{\tau}\left(x_{1}\right)+\varphi^{\tau}\left(x_{1}\right)-\kappa\left|x_{1}^{\tau}\right|^{2}\right)-H_{\epsilon}\left(u_{\tau}\left(x_{1}\right)+\varphi_{\tau}\left(x_{1}\right)\right)\right] \mathcal{F}^{+}\left(w^{\tau}\right)\left(x_{1}\right) \\
& +H_{\epsilon}\left(u_{\tau}\left(x_{1}\right)+\varphi_{\tau}\left(x_{1}\right)\right)\left[\mathcal{F}^{-}\left(w^{\tau}\right)\left(x_{1}\right)-\mathcal{F}^{-}\left(u_{\tau}\right)\left(x_{1}\right)\right] \\
& +\left[1-H_{\epsilon}\left(u_{\tau}\left(x_{1}\right)+\varphi_{\tau}\left(x_{1}\right)\right)\right]\left[\mathcal{F}^{+}\left(w^{\tau}\right)\left(x_{1}\right)-\mathcal{F}^{+}\left(u_{\tau}\right)\left(x_{1}\right)\right] \\
& \leq\left[H_{\epsilon}\left(w^{\tau}\left(x_{1}\right)+\varphi^{\tau}\left(x_{1}\right)-\kappa\left|x_{1}^{\tau}\right|^{2}\right)-H_{\epsilon}\left(u_{\tau}\left(x_{1}\right)+\varphi_{\tau}\left(x_{1}\right)\right)\right]\left[\mathcal{F}^{-}\left(w^{\tau}\right)\left(x_{1}\right)-\mathcal{F}^{+}\left(w^{\tau}\right)\left(x_{1}\right)\right] \\
& +\mathcal{M}^{+}\left(w^{\tau}-u_{\tau}\right)\left(x_{1}\right) .
\end{aligned}
$$

We have obtained

$$
\begin{aligned}
2 \kappa \lambda n & \leq\left[H_{\epsilon}\left(w^{\tau}\left(x_{1}\right)+\varphi^{\tau}\left(x_{1}\right)-\kappa\left|x_{1}^{\tau}\right|^{2}\right)-H_{\epsilon}\left(u_{\tau}\left(x_{1}\right)+\varphi_{\tau}\left(x_{1}\right)\right)\right]\left[\mathcal{F}^{-}\left(w^{\tau}\right)\left(x_{1}\right)-\mathcal{F}^{+}\left(w^{\tau}\right)\left(x_{1}\right)\right] \\
& +\mathcal{M}^{+}\left(w^{\tau}-u_{\tau}\right)\left(x_{1}\right)
\end{aligned}
$$

Now, by (5.9) and (5.10) we have that $\varphi^{\tau}\left(x_{1}\right), \varphi_{\tau}\left(x_{1}\right) \rightarrow 0$ as $\tau \rightarrow 0$. This, combined with (5.16), yields

$$
w^{\tau}\left(x_{1}\right)+\varphi^{\tau}\left(x_{1}\right)-\kappa\left|x_{1}^{\tau}\right|^{2}>u_{\tau}\left(x_{1}\right)+\varphi_{\tau}\left(x_{1}\right)
$$

for $s, \kappa, \tau$ small enough. Since $H_{\epsilon}$ is non-decreasing and $\mathcal{F}^{-}\left(w^{\tau}\right)\left(x_{1}\right)-\mathcal{F}^{+}\left(w^{\tau}\right)\left(x_{1}\right) \leq 0$, we infer that

$$
\left[H_{\epsilon}\left(w^{\tau}\left(x_{1}\right)+\varphi^{\tau}\left(x_{1}\right)-\kappa\left|x_{1}^{\tau}\right|^{2}\right)-H_{\epsilon}\left(u_{\tau}\left(x_{1}\right)+\varphi_{\tau}\left(x_{1}\right)\right)\right]\left[\mathcal{F}^{-}\left(w^{\tau}\right)\left(x_{1}\right)-\mathcal{F}^{+}\left(w^{\tau}\right)\left(x_{1}\right)\right] \leq 0 .
$$

Next, from (5.15),

$$
\mathcal{M}^{+}\left(w^{\tau}-u_{\tau}\right)\left(x_{1}\right) \leq 2 \operatorname{sn} \Lambda .
$$

Plugging the last two inequalities into (5.17), we obtain

$$
2 \kappa \lambda n \leq 2 s n \Lambda,
$$

which is a contradiction for $s$ small enough $(s<\kappa \lambda / \Lambda)$.

We have proven that for any $\delta>0$,

$$
\sup _{x, x+h \sigma \in \Omega} \frac{u^{\epsilon}(x+h \sigma)-u^{\epsilon}(x)}{h} \leq C_{0}+\delta .
$$

Letting $\delta$ go to 0 , we get (5.4).

Note that comparing $u^{\epsilon}$ with the sub and supersolution introduced in Claim 1, we infer that there exists $C_{1}>0$ independent of $\epsilon$ such that

$$
\left\|u^{\epsilon}\right\|_{L^{\infty}(\Omega)} \leq C_{1} .
$$

This bound combined with (5.4) yields a uniform in $\epsilon$ estimate of the Lipschitz norm of the solution $u^{\epsilon}$ of (5.3). Thus the theorem is proven. 
Theorem 5.3. Under the assumptions of Theorem 5.2, there exists a continuous viscosity solution $u^{\epsilon}$ of the $\epsilon$-problem (5.3). Moreover,

$$
\mathcal{F}^{-}\left(u^{\epsilon}\right) \leq 0 \leq \mathcal{F}^{+}\left(u^{\epsilon}\right)
$$

in the viscosity sense in $\Omega$.

Proof. We fix $\epsilon>0$. For $\alpha \in(1 / 2,1)$, let $\Theta:=C^{0, \alpha}(\bar{\Omega})$ be the Banach space of $\alpha$-Hölder continuous functions defined on $\bar{\Omega}$. Let $T$ be the operator defined in the following way, for $u \in \Theta$,

if $v$ is the viscosity solution of

$$
T(u)=v
$$

$$
\begin{cases}H_{\epsilon}(u) \mathcal{F}^{-}(v)+\left(1-H_{\epsilon}(u)\right) \mathcal{F}^{+}(v)=0 & \text { in } \Omega \\ v=f & \text { on } \partial \Omega .\end{cases}
$$

Note that $T$ is well defined. Indeed by Proposition 2.1 the operator $G_{\epsilon}(x, v):=H_{\epsilon}(u) \mathcal{F}^{-}(v)+$ $\left(1-H_{\epsilon}(u)\right) \mathcal{F}^{+}(v)$ is uniformly elliptic. Moreover, since $u \in C^{0, \alpha}(\bar{\Omega})$ with $\alpha>1 / 2, G_{\epsilon}(x, v)$ satisfies the comparison principle, see [14, Theorem III.1]. Let $\psi$ and $\varphi$ be the solutions of

$$
\left\{\begin{array} { l l } 
{ \mathcal { M } ^ { - } ( \psi ) = 0 } & { \text { in } \Omega } \\
{ \psi = f } & { \text { on } \partial \Omega }
\end{array} \text { and } \left\{\begin{array}{ll}
\mathcal{M}^{+}(\varphi)=0 & \text { in } \Omega \\
\varphi=f & \text { on } \partial \Omega
\end{array}\right.\right.
$$

Then, $\psi$ and $\varphi$ are respectively sub and supersolution of (5.19). Thus, by the Perron's method, there exists a unique viscosity solution of (5.19).

Observe that if $T$ has a fixed point $u^{\epsilon}$, that is $T\left(u^{\epsilon}\right)=u^{\epsilon}$, then $u^{\epsilon}$ is solution to (5.3). Moreover, by Remark 5.1. (5.18) also follows. We will prove that we can apply the LeraySchauder fixed point theorem [12, Theorem 11.3] and conclude that $T$ has a fixed point, which concludes the proof. We have:

(1) $T(\Theta) \subset \Theta$ : Let $v=T(u)$, then regularity theory implies that $v \in C^{0, \beta}(\bar{\Omega})$, for any $\beta \in(0,1)$, see [14, Theorem VII.1]. In particular $v \in \Theta$.

(2) $T$ is continuous: Let $\left\{u_{n}\right\} \subset \Theta$ be such that $u_{n} \rightarrow \bar{u}$ in $\Theta$. We need to prove that $v_{n}:=T\left(u_{n}\right) \rightarrow \bar{v}:=T(\bar{u})$ in $\Theta$. By the Hölder estimates for the solutions $v_{n}$, [14, Theorem VII.1], we have that $\left\|v_{n}\right\|_{C^{0, \beta}(\bar{\Omega})} \leq C$ for $\beta>\alpha$. Since the subset of $\Theta$ of $\beta$-Hölder continuous functions on $\bar{\Omega}$ is precompact in $\Theta$, we can extract from $\left\{v_{n}\right\}$ a convergent subsequence. Let $\left\{v_{n_{k}}\right\}$ be any convergent subsequence, $v_{n_{k}} \rightarrow w$ as $k \rightarrow+\infty$ in $\Theta$, then by the stability of viscosity solutions under uniform convergence, it follows that $w$ solves (5.19) with $u=\bar{u}$, that is $w=T(\bar{u})=\bar{v}$. Since every convergent subsequence converges to the same limit function $\bar{v}$, we have that the full sequence $\left\{v_{n}\right\}$ converges to $\bar{v}$ in $\Theta$.

(3) $T$ is compact: By the Hölder estimates, T maps bounded set of $\Theta$ into bounded sets of $C^{0, \beta}(\bar{\Omega}), \beta>\alpha$ which are precompact in $\Theta$.

(4) There exists $M>0$ such that $\|u\|_{\Theta}<M$ for all $u \in \Theta$ and $\sigma \in[0,1]$ satisfying $u=\sigma T(u)$ : the equation $u=\sigma T(u)$ is equivalent to the Dirichlet problem

$$
\begin{cases}H_{\epsilon}(u) \mathcal{F}^{-}(u)+\left(1-H_{\epsilon}(u)\right) \mathcal{F}^{+}(u)=0 & \text { in } \Omega \\ u=\sigma f & \text { on } \partial \Omega .\end{cases}
$$

The estimate $\|u\|_{\Theta}<M$, for some $M>0$, then follows from Theorem 5.2. 
This concludes the proof of the existence of a fixed point $u^{\epsilon}$ and thus of a solution of (5.3) satisfying (5.18).

5.1. Proof of Theorem 3.1. By Theorem 5.3, for any $\epsilon>0$ there exists $u^{\epsilon}$ viscosity solution of (5.3), satisfying also (5.18). By Theorem 5.2 the sequence $\left\{u^{\epsilon}\right\}$ is uniformly Lipschitz continuous, thus by the Ascoli-Arzelà Theorem there exists a subsequence of $\left\{u^{\epsilon}\right\}$ uniformly convergent to a Lipschitz function $u$ solution to (1.4).

If $f^{+}, f^{-} \not \equiv 0$, then by the Lipschitz regularity of $u$ up to the boundary of $\Omega$, we have $u_{1}=u^{+} \not \equiv 0$ and $u_{2}=u^{-} \not \equiv 0$.

\section{Non-Degeneracy at REgular Points}

In this section we introduce the definition of regular points for $u$ solution of (1.4). These are points where at least one among $u_{1}$ and $u_{2}$ has linear growth away from the free boundary, where here and throughout this section we will use the notation introduced in (2.7).

Definition 6.1. Let $u$ be a solution of problem (1.4). Consider $x_{0}$ a point on the free boundary $\Gamma$. We say that $x_{0}$ is a regular point if there exist positive constants $\tilde{r}=\tilde{r}\left(x_{0}\right)$ and $M=M\left(x_{0}\right)$ such that

$$
\sup _{B_{r}\left(x_{0}\right)} U \geq M r
$$

for every $0<r<\tilde{r}$, where

$$
U(x):=\max \left\{u_{1}(x), u_{2}(x)\right\}=|u(x)| .
$$

Otherwise, we say that $x_{0}$ is a singular point.

Lemma 6.2. Let $u$ be a solution of problem (1.4). If $\Gamma$ has a ball from one side at $x_{0} \in \Gamma$, that is there exists a ball $B_{r_{0}}(y)$ contained inside the support of either $u_{1}$ or $u_{2}$, such that $x_{0} \in \partial B_{r_{0}}(y)$, then $x_{0}$ is a regular point.

Proof. Indeed, suppose, without loss of generality, that $B_{r_{0}}(y) \subset \Omega\left(u_{1}\right)$. By (1.4) $u_{1}$ is solution of $\mathcal{F}^{-}\left(u_{1}\right)=0$ in $B_{r_{0}}(y)$. Then by the Harnack inequality we have that $u_{1}(x) \geq M_{1}$, for any $x \in \bar{B}_{\frac{r_{0}}{2}}(y)$ with $M_{1}=C u_{1}(y)$, where $C$ is a universal constant. Let $\phi$ be the solution of

$$
\begin{cases}\mathcal{M}^{-}(\phi)=0 & \text { in } B_{r_{0}}(y) \backslash B_{\frac{r_{0}}{2}}(y) \\ \phi=M_{1} & \text { on } \partial B_{\frac{r_{0}}{2}}(y) \\ \phi=0 & \text { in } \partial B_{r_{0}}(y)\end{cases}
$$

that is, $\phi(x)=M_{1} \frac{1}{2^{\gamma}-1}\left(\frac{r_{0}^{\gamma}}{|x-y|^{\gamma}}-1\right)$, where $\gamma=\Lambda(n-1) / \lambda-1$ and $\lambda$ and $\Lambda$ are the elliptic constants of the Pucci's operator $\mathcal{F}^{-}$(see Lemma 9.1 in Appendix). Then, since in $B_{r_{0}}(y) \backslash$ $B_{\frac{r_{0}}{2}}(y)$

$$
\mathcal{F}^{-}(\phi) \geq \mathcal{M}^{-}(\phi)=0=\mathcal{F}^{-}\left(u_{1}\right)
$$

and $u_{1} \geq \phi$ on $\partial B_{r_{0}}(y) \cup \partial B_{r_{0} / 2}(y)$, the comparison principle and (iii) of Lemma 9.1 imply that for any $x \in B_{r_{0}}(y) \backslash B_{\frac{r_{0}}{2}}(y)$,

$$
u_{1}(x) \geq \phi(x) \geq \frac{M_{1} \gamma}{r_{0}\left(2^{\gamma}-1\right)} d\left(x, \partial B_{r_{0}}(y)\right) .
$$


Hence, for any $r<\frac{r_{0}}{2}$,

$$
\sup _{B_{r}\left(x_{0}\right)} u_{1} \geq \frac{M_{1} \gamma}{r_{0}\left(2^{\gamma}-1\right)} r=: M r .
$$

Therefore, 6.1) holds with $\tilde{r}\left(x_{0}\right)=\frac{r_{0}}{2}$ and

$$
M=\frac{u_{1}(y) C \gamma}{r_{0}\left(2^{\gamma}-1\right)}
$$

depending only on $x_{0}, n, \lambda$ and $\Lambda$.

Remark 6.3. The set of regular points is dense in $\Gamma$. Indeed, since $\Omega\left(u_{1}\right)$ is an open set, the set of points in $\Gamma$ with an interior tangent ball is dense in $\Gamma$. To see it, let $x$ be any point in $\Gamma$. Let us consider a sequence of points $\left\{x_{k}\right\}$ contained in $\Omega\left(u_{1}\right)$ and converging to $x$ as $k \rightarrow \infty$. Let $d_{k}$ be the distance of $x_{k}$ from $\Gamma$. Then the balls $B_{d_{k}}\left(x_{k}\right)$ are contained in $\Omega\left(u_{1}\right)$ and there exist points $y_{k} \in \Gamma \cap \partial B_{d_{k}}\left(x_{k}\right)$ where the $x_{k}$ 's realize the distance from $\Gamma$. The sequence $\left\{y_{k}\right\} \subset \Gamma$ is a sequence of points that have a tangent ball from the inside and converges to $x$.

The following lemma states that at regular points both functions $u_{1}$ and $u_{2}$ have linear growth away from the free boundary.

Lemma 6.4. Let $u$ be a Lipschitz solution of problem (1.4) and let $z \in \Gamma$ be a regular point. Then, there exist $c=c(z)$ and $C$ positive constants such that, for any $0<r<\tilde{r}(z)$,

$$
c r \leq \sup _{B_{r}(z)} u_{i} \leq C r \quad i=1,2
$$

Proof. The inequality $\sup _{B_{r}(z)} u_{i} \leq C r$ for $i=1,2$ follows from the Lipschitz regularity of $u$. We prove that if (6.1) holds true for $x_{0}=z$, then

$$
\sup _{B_{r}(z)} u_{i}(x) \geq c r \quad \text { for any } 0<r<\tilde{r}(z)<d(z, \partial \Omega), \quad i=1,2,
$$

for some $c=c(M)$. Assume by contradiction that for $\epsilon<\frac{M}{4}$ there exists $0<\rho<\tilde{r}$ such that, w.l.o.g.

$$
\sup _{B_{\rho}(z)} u_{2}<\epsilon \rho .
$$

Set $r_{\rho}:=\frac{\rho}{4}<\tilde{r}$. From (6.3) we have that $\sup _{B_{r_{\rho}}(z)} u_{2} \leq \sup _{B_{\rho}(z)} u_{2}<\epsilon \rho$ and hence

$$
\sup _{B_{r_{\rho}}(z)} u_{2}<(4 \epsilon) \frac{\rho}{4}<M \frac{\rho}{4}=M r_{\rho} .
$$

Therefore from (6.4) and the fact that $\sup _{B_{r_{\rho}}(z)} U \geq M r_{\rho}$, where $U=\max \left\{u_{1}, u_{2}\right\}$, we must have

$$
\sup _{B_{r_{\rho}}(z)} u_{1} \geq M r_{\rho}
$$

that is, there exists $y \in \bar{B}_{r_{\rho}}(z)$ such that

$$
u_{1}(y) \geq M r_{\rho}
$$

Consider the ball centered at $y$ with radius $h$, where $h=\left|y-x_{0}\right|$, being $x_{0} \in \Gamma$ the closest point to $y$ in $\Gamma$. Observe that $h \leq \frac{\rho}{4}$. Next, the ball $B_{h}(y)$ is contained in $\Omega\left(u_{1}\right)$, therefore by (1.4) $\mathcal{F}^{-}\left(u_{1}\right)=0$ in $B_{h}(y)$. The Harnack inequality then implies $u_{1} \geq C M r_{\rho}$ on $\bar{B}_{\frac{h}{2}}(y)$, where 
$C$ is a universal constant. Let $\phi$ be the function defined as follows:

$$
\phi(x)=C M \frac{r_{\rho}}{2^{\gamma}-1}\left(\frac{h^{\gamma}}{|x-y|^{\gamma}}-1\right),
$$

with $\gamma=\frac{\Lambda(n-1)-\lambda}{\lambda}$. Then, $\phi$ satisfies

$$
\begin{cases}\mathcal{M}^{-}(\phi)=0 & \text { in } \mathbb{R}^{n} \backslash\{y\} \\ \phi=C M r_{\rho} & \text { on } \partial B_{\frac{h}{2}}(y) \\ \phi=0 & \text { on } \partial B_{h}(y),\end{cases}
$$

see Lemma 9.1. In particular, since $u_{1} \geq \phi$ on $\partial B_{h}(y) \cup \partial B_{\frac{h}{2}}(y)$, by the comparison principle,

$$
u_{1}(x) \geq \phi(x), \quad x \in B_{h}(y) \backslash B_{\frac{h}{2}}(y) .
$$

The previous inequality still holds in the complement of $B_{h}(y)$ in $\Omega\left(u_{1}\right)$, being $\phi$ negative in that set. Therefore, we have that

$$
u(x)=u_{1}(x) \geq \phi(x) \quad \text { if } x \in \Omega\left(u_{1}\right) \backslash B_{\frac{h}{2}}(y) .
$$

To continue the proof, we will prove that $\phi \leq-u_{2}$ in a neighborhood of $x_{0}$ in $\Omega\left(u_{2}\right)$. If $x \in B_{2 h}(y)$ then $d(x, z) \leq d(x, y)+d(y, z) \leq 2 h+r_{\rho} \leq \frac{3}{4} \rho$, therefore $B_{2 h}(y) \subset B_{\rho}(z)$. In particular, by (6.3),

On the other hand,

$$
\sup _{B_{2 h}(y)} u_{2}<\epsilon \rho=\epsilon 4 r_{\rho}
$$

$$
\phi=-\frac{C M r_{\rho}}{2^{\gamma}} \text { on } \partial B_{2 h}(y) \text { and } \phi \leq 0 \text { on } \Gamma .
$$

Let $\epsilon$ be so small that $4 \epsilon \leq \frac{C M}{2 \gamma}$, then

$$
\phi \leq-u_{2} \quad \text { on } \partial\left(\Omega\left(u_{2}\right) \cap B_{2 h}(y)\right) \quad \text { and } \quad \phi<-u_{2} \quad \text { on } \Gamma \cap B_{2 h}(y) .
$$

Since in addition, in the set $\Omega\left(u_{2}\right) \cap B_{2 h}(y)$ we have

$$
\mathcal{F}^{-}(\phi) \geq \mathcal{M}^{-}(\phi)=0 \quad \text { and } \quad \mathcal{F}^{-}\left(-u_{2}\right)=\mathcal{F}^{-}(u) \leq 0,
$$

the strong maximum principle implies

$$
\phi(x)<-u_{2}(x) \text { for any } x \in \Omega\left(u_{2}\right) \cap B_{2 h}(y) .
$$

Putting all together, by (6.6) and the previous inequality, we conclude that for all $x \in B_{\frac{h}{2}}\left(x_{0}\right)$ the function $u=u_{1}-u_{2}$ satisfies

$$
u(x) \geq \phi(x), u \not \equiv \phi \text { and } u\left(x_{0}\right)=\phi\left(x_{0}\right) .
$$

This is in contradiction with the strong maximum principle, since we know that $\mathcal{F}^{-}(u) \leq 0 \leq$ $\mathcal{F}^{-}(\phi)$ in $B_{\frac{h}{2}}\left(x_{0}\right)$. The contradiction has followed by assuming that there exists $0<\rho<\tilde{r}$ such that (6.3) holds true, with $\epsilon$ satisfying $\epsilon<\frac{M}{4}$ and $4 \epsilon \leq \frac{C M}{2^{\gamma}}$. Therefore, if we choose for example

$$
c=\frac{1}{2} \min \left\{\frac{M}{4}, \frac{C M}{2^{\gamma+2}}\right\},
$$

inequalities (6.2) hold true. 
Lemma 6.5. Let $z \in \Gamma$ be a regular point and let $u$ be a Lipschitz solution of problem (1.4). Then there exists a constant $C=C(z)>0$ such that for every $0<r<\tilde{r}$,

$$
J_{r}\left(u_{i}, z\right) \geq C, \quad i=1,2,
$$

where $J_{r}\left(u_{i}, z\right)$ is defined by (2.8).

Proof. Without loss of generality, we prove the lemma for $i=1$. By Lemma 6.4 there exists $c=c(z)>0$ such that for any radius $r<\tilde{r}<d(z, \partial \Omega)$, there exists $y \in \bar{B}_{\frac{r}{4}}(z)$ such that

$$
u_{1}(y) \geq c \frac{r}{4} .
$$

Let $x_{0} \in \Gamma$ be the closest point in the free boundary to $y, h=\left|y-x_{0}\right|$ and consider $B_{h}(y)$. Note that $h \leq|y-z| \leq r / 4$,

$$
B_{h}(y) \subset \Omega\left(u_{1}\right) \cap B_{r}(z)
$$

and $B_{h}\left(x_{0}\right) \subset B_{r}(z)$. Moreover, since $u_{1}$ is Lipschitz continuous in $\Omega$, there exists $L$ such that $|u(x)-u(y)| \leq L|x-y|$ for any $x, y \in B_{r}(z)$. In particular, we have that

$$
c \frac{r}{4} \leq u_{1}(y)-u_{1}\left(x_{0}\right)=u_{1}(y) \leq L\left|y-x_{0}\right|=L h,
$$

which implies

$$
\frac{h}{r} \geq \frac{c}{4 L} .
$$

Next, since (6.9) holds, Lemma 6.2 implies that $x_{0}$ is also a regular point and for any $x \in$ $B_{h}(y) \backslash B_{\frac{h}{2}}(y)$,

$$
u_{1}(x) \geq M d\left(x, \partial B_{h}(y)\right)=M(h-|x-y|),
$$

where $M=\frac{u_{1}(y) C \gamma}{h\left(2^{\gamma}-1\right)}$, (see proof for Lemma 6.2). In particular, for any $s<\frac{h}{2}$,

$$
\sup _{B_{s}\left(x_{0}\right)} u_{1} \geq M s
$$

We now note that, from (6.8) and the inequality $h \leq r / 4$,

$$
M \geq \frac{c \gamma C}{2^{\gamma}-1}=: \tilde{M},
$$

where $\tilde{M}$ depends on $z$ but it is independent of $x_{0}, h$ and $r$. Since now we have, for any $s<h / 2$

$$
\sup _{B_{s}\left(x_{0}\right)} U \geq \tilde{M} s
$$

where $U=\max \left\{u_{1}, u_{2}\right\}$, by Lemma 6.4 there exists $\tilde{c}=\tilde{c}(\tilde{M})$ such that, for any $s<h / 2$,

$$
\sup _{B_{s}\left(x_{0}\right)} u_{2} \geq \tilde{c} s .
$$

We are now in conditions to apply a Poincaré-Sobolev type inequality to $u_{1}$ (see e.g. [13, Chapter 4, Lemma 2.8] and [15, Theorem 3]). Indeed, we claim that the zero set of $u_{1}$ has positive density.

Claim: There exists $\epsilon>0$ independent of $h$ such that

$$
\left|\left\{u_{1}=0\right\} \cap B_{\frac{h}{2}}\left(x_{0}\right)\right| \geq\left|\left\{u_{2}>0\right\} \cap B_{\frac{h}{2}}\left(x_{0}\right)\right| \geq \epsilon\left|B_{\frac{h}{2}}\left(x_{0}\right)\right| .
$$


Proof of the claim: Suppose by contradiction that for any $\epsilon>0$ one has $\left|\left\{u_{2}>0\right\} \cap B_{\frac{h}{2}}\left(x_{0}\right)\right|<$ $\epsilon\left|B_{\frac{h}{2}}\left(x_{0}\right)\right|$. Since $u_{2}$ is Lipschitz continuous in $\Omega$, there exists $L>0$ such that for all $x \in B_{\frac{h}{2}}\left(x_{0}\right)$,

$$
u_{2}(x) \leq L\left|x-x_{0}\right| \leq L \frac{h}{2} .
$$

Since $u_{2}$ is subharmonic, see (4.2), the mean-value Theorem implies that for any $x \in B_{\frac{h}{4}}\left(x_{0}\right)$,

$$
u_{2}(x) \leq \int_{B_{\frac{h}{4}}(x)} u_{2}(t) d t \leq \frac{1}{\left|B_{\frac{h}{4}}(x)\right|} \int_{\left\{u_{2}>0\right\} \cap B_{\frac{h}{2}}\left(x_{0}\right)} u_{2}(t) d t \leq \frac{\epsilon\left|B_{\frac{h}{2}}\left(x_{0}\right)\right|}{\left|B_{\frac{h}{4}}(x)\right|} L \frac{h}{2}=\epsilon 2^{n-1} L h,
$$

which is in contradiction with (6.13) with $s=\frac{h}{4}$ for $\epsilon<\frac{\tilde{c}}{L 2^{n+1}}$. This proves (6.14).

Next, to conclude the proof of the lemma, since

$$
\frac{1}{r^{2}} \int_{B_{r}(z)} \frac{\left|\nabla u_{1}(x)\right|^{2}}{|x-z|^{n-2}} d x \geq \frac{1}{r^{n}} \int_{B_{r}(z)}\left|\nabla u_{1}(x)\right|^{2} d x \geq \frac{1}{r^{n}} \int_{B_{\frac{h}{2}}\left(x_{0}\right)}\left|\nabla u_{1}(x)\right|^{2} d x
$$

we just need to bound from below the last integral.

Since (6.14) holds true, we can apply the Poincaré-Sobolev type inequality to obtain

$$
\frac{1}{r^{n}} \int_{B_{\frac{h}{2}}\left(x_{0}\right)}\left|\nabla u_{1}(x)\right|^{2} d x \geq \frac{1}{r^{n}} \frac{1}{C(n, \epsilon) h^{2}} \int_{B_{\frac{h}{2}}\left(x_{0}\right)} u_{1}^{2}(x) d x .
$$

Finally, by using (6.11) and (6.12), we get

$$
\begin{aligned}
\frac{1}{r^{n}} \frac{1}{C(n, \epsilon) h^{2}} \int_{B_{\frac{h}{2}}\left(x_{0}\right)} u_{1}^{2}(x) d x & \geq \frac{1}{r^{n}} \frac{1}{C(n, \epsilon) h^{2}} \int_{B_{\frac{h}{2}}\left(x_{0}\right) \cap B_{h}(y)} \tilde{M}^{2}(h-|x-y|)^{2} d x \\
& \geq \frac{1}{r^{n}} \frac{1}{C(n, \epsilon) h^{2}} \int_{B_{\frac{h}{2}}\left(x_{0}\right) \cap B_{\frac{7}{8} h}(y)} \tilde{M}^{2}(h-|x-y|)^{2} d x \\
& \geq \frac{\tilde{M}^{2} h^{2} h^{n}}{r^{n} C(n, \epsilon) h^{2}},
\end{aligned}
$$

where in the last inequality we have used that $\left|B_{\frac{h}{2}}\left(x_{0}\right) \cap B_{\frac{7}{8} h}(y)\right| \geq \bar{c} h^{n}$ and $\tilde{M}^{2}(h-|x-y|)^{2} \geq$ $\frac{\tilde{M}^{2}}{64} h^{2}$ for any $x \in B_{\frac{7}{8} h}(y)$.

Putting all together, from (6.10), (6.16), (6.17) and (6.18) we infer that there exists $C=$ $C(z)>0$ such that

and this concludes the proof of the lemma.

$$
\frac{1}{r^{2}} \int_{B_{r}(z)} \frac{\left|\nabla u_{1}(x)\right|^{2}}{|x-z|^{n-2}} d x \geq C
$$

The following is an immediate corollary of Lemma 6.5.

Corollary 6.6. Let $u$ be a Lipschitz solution of problem (1.4) and let $z \in \Gamma$ be a regular point. Then there exists a constant $C=C(z)>0$ such that, for any $0<r<\tilde{r}$,

$$
J_{r}(u, z) \geq C
$$

where $J_{r}(u, z)$ is defined by (2.9). 


\section{Proof of Theorem 3.2}

We start with the analysis of the blow up of the solution at regular points. As in Section 6, throughout this section we will use the notation introduced in (2.7) for $u$ solution of (1.4).

Lemma 7.1. Let $u$ be a Lipschitz solution of problem (1.4). Let $0 \in \Gamma$ be a regular point. Let $u_{r}$ denote the blow-up sequence

$$
u_{r}(x):=\frac{1}{r} u(r x), \quad x \in B_{2}(0),
$$

with $r<d(0, \partial \Omega) / 2$. Then, $u_{r}$ admits a uniformly converging subsequence in $B_{1}(0)$ and for any converging subsequence $u_{r_{j}}(x)=u\left(r_{j} x\right) / r_{j}, j \in \mathbb{N}$, there exist $\alpha, \beta>0$ and a unit vector $n$, such that,

$$
J_{0}(u)=c_{n} \alpha^{2} \beta^{2}
$$

where $J_{0}(u)$ is defined as in (4.3), and as $j \rightarrow+\infty$,

$$
u_{r_{j}}(x) \rightarrow \alpha<x, n>^{+}-\beta<x, n>^{-},
$$

uniformly in $B_{1}(0)$.

Proof. Since $u$ is Lipschitz continuous in $\Omega$, the sequence $\left\{u_{r}\right\}$ is uniformly bounded in $C^{0,1}\left(B_{2}(0)\right)$. Therefore, by Ascoli-Arzela, there exists a subsequence $\left\{u_{r_{j}}\right\}$ and a Lipschitz function $\bar{u}$, such that, as $j \rightarrow+\infty, u_{r_{j}} \rightarrow \bar{u}$ uniformly in $B_{1}(0)$ and weakly in $H^{1}\left(B_{1}(0)\right)$. In particular,

$$
\int_{B_{1}(0)}|\nabla \bar{u}|^{2} d x \leq \liminf _{j \rightarrow+\infty} \int_{B_{1}(0)}\left|\nabla u_{r_{j}}\right|^{2} d x
$$

We will prove that for any $s \in(0,1)$,

$$
J_{s}(\bar{u})=J_{0}(u)>0
$$

where $J_{r}(u)$ is defined as in (2.9). For that, we truncate $\left(u_{1}\right)_{r_{j}}=u_{1}\left(r_{j} x\right) / r_{j}$ at level $\epsilon$ and $h$, for $0<\epsilon<h$, by considering $w_{\epsilon, h}:=\min \left\{\max \left\{\left(u_{1}\right)_{r_{j}}, \epsilon\right\}, h\right\}$. Since each $\left(u_{1}\right)_{r_{j}}$ is subharmonic (i.e., $\Delta\left(u_{1}\right)_{r_{j}}=\mu_{r_{j}} \geq 0$, in the sense of distributions, and $\mu_{j}$ is a Radon measure) and Lipschitz continuous, then we have $w_{\epsilon, h} \nabla\left(u_{1}\right)_{r_{j}} \in L^{\infty}\left(B_{1}(0)\right)$ and the product rule $\operatorname{div}\left(w_{\epsilon, h} \nabla\left(u_{1}\right)_{r_{j}}\right)=$ $w_{\epsilon, h} \mu_{r_{j}}+\nabla w_{\epsilon, h} \cdot \nabla\left(u_{1}\right)_{r_{j}}$ holds in the sense of distributions. Moreover, we can integrate by parts (see [7, 8] ) in $B_{1}(0)$ :

$$
\int_{B_{1}(0)} \operatorname{div}\left(w_{\epsilon, h} \nabla\left(u_{1}\right)_{r_{j}}\right)=\int_{\partial B_{1}(0)}\left(w_{\epsilon, h} \nabla\left(u_{1}\right)_{r_{j}} \cdot \nu\right)_{t r} d \mathcal{H}^{n-1},
$$

where $\left(w_{\epsilon, h} \nabla\left(u_{1}\right)_{r_{j}} \cdot \nu\right)_{t r} \in L^{\infty}\left(\partial B_{1}(0)\right)$ is the normal trace of the vector field $w_{\epsilon, h} \nabla\left(u_{1}\right)_{r_{j}}$ and which satisfies

$$
\left(w_{\epsilon, h} \nabla\left(u_{1}\right)_{r_{j}} \cdot \nu\right)_{t r} \leq\left\|w_{\epsilon, h} \nabla\left(u_{1}\right)_{r_{j}}\right\|_{L^{\infty}\left(B_{1}(0)\right)} \leq h\left\|\nabla\left(u_{1}\right)_{r_{j}}\right\|_{L^{\infty}\left(B_{1}(0)\right)} \leq h L .
$$

From (7.5), and since $\nabla w_{\epsilon, h}=0$ a.e. in $B_{1}(0) \cap\left\{\left(u_{1}\right)_{r_{j}} \geq h\right\}$ and in $B_{1}(0) \cap\left\{\left(u_{1}\right)_{r_{j}} \leq \epsilon\right\}$, we obtain

$$
\begin{aligned}
0 & \leq \int_{B_{1}(0)} w_{\epsilon, h} d \mu_{r_{j}}=-\int_{\left.\epsilon \leq\left(u_{1}\right)_{r_{j}} \leq h\right\} \cap B_{1}(0)}\left|\nabla\left(u_{1}\right)_{r_{j}}\right|^{2} d x+\int_{\partial B_{1}(0)}\left(w_{\epsilon, h} \nabla\left(u_{1}\right)_{r_{j}} \cdot \nu\right)_{t r} d \mathcal{H}^{n-1} \\
& \leq-\int_{\left\{\epsilon \leq\left(u_{1}\right)_{r_{j}} \leq h\right\} \cap B_{1}(0)}\left|\nabla\left(u_{1}\right)_{r_{j}}\right|^{2} d x+C h,
\end{aligned}
$$


with $C=L \mathcal{H}^{n-1}\left(\partial B_{1}(0)\right)$. Using the Lebesgue Dominated Convergence Theorem we let $\epsilon$ go to 0 , obtaining:

$$
\int_{\left\{0 \leq\left(u_{1}\right)_{r_{j}} \leq h\right\} \cap B_{1}(0)}\left|\nabla\left(u_{1}\right)_{r_{j}}\right|^{2} d x \leq C h .
$$

Similarly, one gets

$$
\int_{\left\{0 \leq\left(u_{2}\right)_{r_{j}} \leq h\right\} \cap B_{1}(0)}\left|\nabla\left(u_{2}\right)_{r_{j}}\right|^{2} d x \leq C h .
$$

From (7.8) and (7.9), we obtain

$$
\int_{\left\{\left|u_{r_{j}}\right| \leq h\right\} \cap B_{1}(0)}\left|\nabla u_{r_{j}}\right|^{2} d x \leq C h .
$$

Next, for $j$ large enough, the set $\left\{\left|u_{r_{j}}\right|>0\right\}$ contains $\{|\bar{u}|>h\}$. Moreover, since $u_{r_{j}}$ is a Lipschitz viscosity solution of

$$
\begin{cases}\mathcal{F}^{-}\left(u_{r_{j}}\right)=0 & \text { in }\left\{u_{r_{j}}>0\right\} \cap B_{2}(0) \\ \mathcal{F}^{+}\left(u_{r_{j}}\right)=0 & \text { in }\left\{u_{r_{j}}<0\right\} \cap B_{2}(0),\end{cases}
$$

by the interior $C^{2, \alpha}$ estimates for the operators $\mathcal{F}^{ \pm}$(see Remark 2.3), up to a subsequence, $\nabla u_{r_{j}} \rightarrow \nabla \bar{u}$ uniformly in $\{|\bar{u}|>h\} \cap B_{1}(0)$ as $j \rightarrow+\infty$, and thus,

$$
\lim _{j \rightarrow+\infty} \int_{\left\{\left|u_{r_{j}}\right|>h\right\} \cap B_{1}(0)}\left|\nabla u_{r_{j}}\right|^{2} d x=\int_{\{|\bar{u}|>h\} \cap B_{1}(0)}|\nabla \bar{u}|^{2} d x .
$$

By (7.10) and (7.12) we infer that, for any $h>0$,

$$
\limsup _{j \rightarrow+\infty} \int_{B_{1}(0)}\left|\nabla u_{r_{j}}\right|^{2} d x \leq \int_{B_{1}(0)}|\nabla \bar{u}|^{2} d x+C h,
$$

which combined with (17.3) yields, letting $h \rightarrow 0$,

$$
\lim _{j \rightarrow+\infty} \int_{B_{1}(0)}\left|\nabla u_{r_{j}}\right|^{2} d x=\int_{B_{1}(0)}|\nabla \bar{u}|^{2} d x .
$$

By (7.13),$\left|\nabla u_{r_{j}}\right|^{2} \rightarrow|\nabla \bar{u}|^{2}$ a.e. in $B_{1}(0)$. Since in addition $\left|\nabla u_{r_{j}}\right|^{2} /|x|^{n-2} \leq L^{2} /|x|^{n-2} \in$ $L^{1}\left(B_{1}(0)\right)$, by the Dominated Convergence Theorem we infer that, for any $s \in(0,1)$,

$$
\lim _{j \rightarrow+\infty} J_{s}\left(u_{r_{j}}\right)=J_{s}(\bar{u}) .
$$

Next, by Corollary 6.6 and Remark 4.2

$$
\lim _{r \rightarrow 0^{+}} J_{r}(u)=J_{0}(u)>0 .
$$

Let $s \in(0,1)$. A change of variables yields:

$$
J_{s}\left(u_{r_{j}}\right)=J_{s r_{j}}(u) .
$$

Therefore, by (7.14)-(7.16), for any $s \in(0,1)$,

$$
J_{s}(\bar{u})=\lim _{j \rightarrow+\infty} J_{s}\left(u_{r_{j}}\right)=\lim _{j \rightarrow+\infty} J_{s r_{j}}(u)=J_{0}(u)>0,
$$

which gives (7.4). The conclusion of the lemma follows from Theorem 4.3,

Corollary 7.2. Under the assumptions of Lemma 7.1, for any $\epsilon>0$ there exists $J \in \mathbb{N}$ such that for any $j \geq J$, all the level sets of $u_{r_{j}}$ in $B_{1}(0)$ are $\epsilon$-flat, in the sense that for any $\lambda \in \mathbb{R}$, 
we have

$$
\left\{u_{r_{j}}=\lambda\right\} \cap B_{1}(0) \subset\left\{x \in \mathbb{R}^{n} \mid d\left(x, \Pi_{\lambda}\right)<c \epsilon\right\},
$$

for some $c>0$ independent of $\lambda, r_{j}$ and $\epsilon$, where

$$
\Pi_{\lambda}= \begin{cases}\{<x, n>=\lambda / \alpha\} & \text { if } \lambda>\epsilon, \\ \{<x, n>=0\} & \text { if } \lambda \in[-\epsilon, \epsilon], \\ \{<x, n>=\lambda / \beta\} & \text { if } \lambda<-\epsilon .\end{cases}
$$

and $n, \alpha$ and $\beta$ are as in (7.2).

Proof. By Lemma 7.1, for any $\epsilon>0$, there exists $J \in \mathbb{N}$ such that for any $j \geq J$,

$$
\left|u_{r_{j}}(x)-\alpha<x, n>^{+}+\beta<x, n>^{-}\right|<\frac{\epsilon}{2},
$$

for all $x \in B_{1}(0)$. Let $\left.\lambda\right\rangle \epsilon$, then by (17.18), if $\langle x, n\rangle \geq \frac{\lambda+\epsilon}{\alpha}$ then

$$
u_{r_{j}}(x) \geq \lambda+\epsilon-\frac{\epsilon}{2}=\lambda+\frac{\epsilon}{2}>\lambda,
$$

and if $\langle x, n\rangle \leq \frac{\lambda-\epsilon}{\alpha}$, then

We infer that

$$
u_{r_{j}}(x) \leq \lambda-\frac{\epsilon}{2}<\lambda .
$$

$$
\left\{u_{r_{j}}=\lambda\right\} \cap B_{1}(0) \subset\left\{x \in \mathbb{R}^{n} \mid \frac{-\epsilon}{\alpha} \leq<x, n>-\frac{\lambda}{\alpha} \leq \frac{\epsilon}{\alpha}\right\} .
$$

Similarly, if $\lambda \in[-\epsilon, \epsilon]$,

$$
\left\{u_{r_{j}}=\lambda\right\} \cap B_{1}(0) \subset\left\{x \in \mathbb{R}^{n} \mid \frac{-2 \epsilon}{\beta} \leq<x, n>\leq \frac{2 \epsilon}{\alpha}\right\},
$$

and if $\lambda<-\epsilon$,

$$
\left\{u_{r_{j}}=\lambda\right\} \cap B_{1}(0) \subset\left\{x \in \mathbb{R}^{n} \mid \frac{-\epsilon}{\beta} \leq<x, n>-\frac{\lambda}{\beta} \leq \frac{\epsilon}{\beta}\right\} .
$$

Inclusions (7.19), (7.20) and (7.21) give (7.17) with

$$
c=2 \max \left\{\alpha^{-1}, \beta^{-1}\right\} .
$$

By Lemma 7.1 we know that if $0 \in \Gamma$ is a regular point, then the blow up sequence $u(r x) / r$ admits a subsequence converging to a two-plane solution of the form (17.2). We next show that if there is a tangent ball from one side to $\Gamma$ at 0 , then the full sequence $u(r x) / r$ converges to a two-plane solution and therefore $u$ has an asymptotic linear behavior at 0 .

Lemma 7.3. Let $u$ be a Lipschitz solution of problem (1.4). Let $0 \in \Gamma$. Assume that there exists a tangent ball $B$ from one side to $\Gamma$ at 0 . Then, there exist $\alpha, \beta>0$ such that

$$
\left.u(x)=\alpha<x, \nu\rangle^{+}-\beta<x, \nu\right\rangle^{-}+o(|x|),
$$

where $\nu$ is the normal vector of $B$ at 0 pointing inward $\{u>0\}$.

Proof. By Lemma 6.2, 0 is a regular point. Consider the sequence $u_{r}(x)=\frac{1}{r} u(r x)$, for $r$ small enough. By Lemma 7.1] there exist a subsequence $\left\{u_{r_{j}}\right\}$, a unit vector $n$ and $\alpha, \beta$ positive constants satisfying (7.1), such that as $j \rightarrow+\infty$,

$$
u_{r_{j}}(x) \rightarrow \bar{u}(x):=\alpha<x, n>^{+}-\beta<x, n>^{-},
$$


uniformly in $B_{1}(0)$.

Assume, without loss of generality, that there exists a ball $B_{r_{0}}(y) \subset \Omega\left(u_{1}\right)$ such that $0 \in$ $\partial B_{r_{0}}(y)$. Let $\nu$ be the normal vector of $B_{r_{0}}(y)$ at 0 pointing inward $\Omega\left(u_{1}\right)$.

Claim 1: We claim that $\nu=n$.

Proof of Claim 1 : Indeed, suppose by contradiction that $\nu \neq n$. Then, there exists $x_{0} \in B_{1}(0)$ such that for any $j,\left\langle r_{j} x_{0}, \nu>>0\right.$ and $<r_{j} x_{0}, n><0$. Fix $J \in \mathbb{N}$ such that the sequence of points $\left\{r_{j} x_{0}\right\}$ satisfies $r_{j} x_{0} \in B_{r_{0}}(y) \subset \Omega\left(u_{1}\right)$ for all $j \geq J$, then

$$
\frac{u\left(r_{j} x_{0}\right)}{r_{j}}>0 \text { for all } j \geq J
$$

Passing to the limit as $j \rightarrow+\infty$, we get

$$
\bar{u}\left(x_{0}\right) \geq 0 .
$$

On the other hand,

$$
\bar{u}\left(x_{0}\right)=-\beta<x_{0}, n>^{-}<0 .
$$

This is a contradiction. Therefore we must have $\nu=n$.

We now proceed to show that the full sequence $u_{r}$ converges to $\bar{u}$. Let $\bar{u}$ and $\bar{v}$ be the limits of two converging subsequences of the sequence $\left\{u_{r}\right\}$, then we must have

$$
\bar{u}=\alpha_{1}\langle x, \nu\rangle^{+}-\beta_{1}\langle x, \nu\rangle^{-}
$$

and

$$
\bar{v}=\alpha_{2}<x, \nu>^{+}-\beta_{2}\langle x, \nu\rangle^{-} .
$$

Claim 2: We claim that in addition that

$$
\alpha_{1}=\alpha_{2} \text { and } \beta_{1}=\beta_{2} .
$$

Proof of Claim 2: To prove this claim, we will construct a barrier to bound $u_{1}$ from below. Let $\phi$ be the solution of

$$
\begin{cases}\mathcal{F}^{-}(\phi)=0 & \text { in } B_{r_{0}}(y) \backslash B_{\frac{r_{0}}{2}}(y) \\ \phi=1 & \text { on } \partial B_{\frac{r_{0}}{2}}(y) \\ \phi=0 & \text { on } \partial B_{r_{0}}(y) .\end{cases}
$$

By the comparison principle, for any $x \in B_{r_{0}}(y) \backslash B_{\frac{r_{0}}{2}}(y)$

$$
u_{1}(x) \geq c_{0} \phi(x), \quad \text { with } c_{0}=\min _{\partial B_{\frac{r_{0}}{2}}(y)} u_{1} .
$$

For $k \geq 0$ such that $2^{-k}<r_{0} / 2$, let

$$
\tilde{m}_{k}:=\sup \left\{m \mid u_{1}(x) \geq m \phi(x) \text { in } B_{2^{-k}}(0) \cap B_{r_{0}}(y)\right\} .
$$

Notice that the sequence $\tilde{m}_{k}$ is increasing. Moreover, by (17.24), $\tilde{m}_{k} \geq c_{0}$ for any $k$. Let

$$
\tilde{m}_{\infty}:=\sup _{k} \tilde{m}_{k}=\lim _{k \rightarrow+\infty} \tilde{m}_{k} .
$$

Since $u_{1}$ is Lipschitz continuous, $\tilde{m}_{\infty}<+\infty$.

By Lemma 9.2, there exists $\sigma>0$ independent of $r_{0}$ such that, for any $x \in B_{r_{0}}(y) \backslash B_{\frac{r_{0}}{2}}(y)$,

$$
\phi(x)=\frac{\sigma}{r_{0}}<x, \nu>+o(|x|) .
$$

Set

$$
m_{k}:=\frac{\sigma}{r_{0}} \tilde{m}_{k} \quad \text { and } \quad m_{\infty}:=\frac{\sigma}{r_{0}} \tilde{m}_{\infty}
$$


By the definition of $m_{k}$ and (7.25), for $x \in B_{2^{-k}}(0) \cap B_{r_{0}}(y)$ we have

$$
u_{1}(x) \geq \tilde{m}_{k} \phi(x)=m_{k}<x, \nu>+o(|x|) .
$$

This implies that $\alpha_{1} \geq m_{\infty}$. Assume by contradiction that $\alpha_{1}>m_{\infty}$. We will show that in this case, there exists $\epsilon>0$ and a sequence $k_{j}$ such that, for $j$ large enough,

$$
u_{1}-\left(\tilde{m}_{k_{j}}+\epsilon\right) \phi(x) \geq 0 \text {, for all } x \in B_{2}-k_{j}(0) \cap B_{r_{0}}(y),
$$

which is in contradiction with the definition of $\tilde{m}_{k_{j}}$. For that, if $\alpha_{1}>m_{\infty}$, there exists a sequence $r_{j} \rightarrow 0$ as $j \rightarrow+\infty$ such that if $y_{j}=r_{j} \nu$, then, for some $\delta_{0}>0$,

$$
u_{1}\left(y_{j}\right)-\tilde{m}_{\infty} \phi\left(y_{j}\right) \geq \delta_{0} r_{j} .
$$

Let $\tilde{m}_{k_{j}}$ be a subsequence converging to $\tilde{m}_{\infty}$ as $j \rightarrow+\infty$ such that, up to eventually consider a subsequence of $\left\{r_{j}\right\}$, one has that $2 r_{j} \leq 2^{-k_{j}}$. Then $B_{r_{j}}\left(y_{j}\right) \subset B_{2^{-k_{j}}}(0) \cap B_{r_{0}}(y)$ and since $\tilde{m}_{k_{j}} \leq \tilde{m}_{\infty}$

$$
u_{1}\left(y_{j}\right)-\tilde{m}_{k_{j}} \phi\left(y_{j}\right) \geq \delta_{0} r_{j} .
$$

By the definition of $\tilde{m}_{k_{j}}$ the function $u_{1}-\tilde{m}_{k_{j}} \phi$ is positive in $B_{2^{-k_{j}}}(0) \cap B_{r_{0}}(y)$ and by Proposition 2.1 it satisfies

$$
\mathcal{M}^{-}\left(u_{1}-\tilde{m}_{k_{j}} \phi\right) \leq \mathcal{F}^{-}\left(u_{1}-\tilde{m}_{k_{j}} \phi\right) \leq \mathcal{F}^{-}\left(u_{1}\right)-\mathcal{F}^{-}\left(\tilde{m}_{k_{j}} \phi\right)=0
$$

and

$$
\mathcal{M}^{+}\left(u_{1}-\tilde{m}_{k_{j}} \phi\right) \geq \mathcal{F}^{-}\left(u_{1}\right)-\mathcal{F}^{-}\left(\tilde{m}_{k_{j}} \phi\right)=0 .
$$

Therefore, since $B_{r_{j}}\left(y_{j}\right) \subset B_{2^{-k_{j}}}(0) \cap B_{r_{0}}(y)$, by the Harnack inequality,

$$
u_{1}(x)-\tilde{m}_{k_{j}} \phi(x) \geq c \delta_{0} r_{j} \quad \text { for } x \in B_{\frac{r_{j}}{2}}\left(y_{j}\right),
$$

where $c>0$ is a universal constant. By a barrier argument we see that there exist $\delta_{j}$ and $\tilde{c}>0$ (independent of $j$ ) such that:

$$
u_{1}(x)-\tilde{m}_{k_{j}} \phi(x) \geq \tilde{c} d\left(x, \partial B_{r_{0}}(y)\right) \quad \text { for } x \in B_{\delta_{j}}(0) \cap B_{r_{0}}(y) .
$$

Indeed, let $z \in B_{\frac{r_{j}}{4}}\left(y_{j}\right) \cap \partial B_{r_{0}-r_{j}}(y)$ and let $w$ be the closest point to $z$ in $\partial B_{r_{0}}(y)$, that is $w \in \partial B_{r_{0}}(y) \cap \partial B_{r_{j}}(z)$. By (1.27),$u_{1}(x)-\tilde{m}_{k_{j}} \phi(x) \geq c \delta_{0} r_{j}$ for $x \in B_{\frac{r_{j}}{4}}(z)$. Let $\psi(x)$ be the solution to

$$
\begin{cases}\mathcal{M}^{-}(\psi)=0 & \text { in } B_{r_{j}}(z) \backslash B_{\frac{r_{j}}{4}}(z) \\ \psi=c \delta_{0} r_{j} & \text { on } \partial B_{\frac{r_{j}}{4}}(z) \\ \psi=0 & \text { on } \partial B_{r_{j}}(z) .\end{cases}
$$

By Lemma 9.1, $\psi(x)=\frac{c \delta_{0} r_{j}}{4^{\gamma}-1}\left(\frac{r_{j}^{\gamma}}{|x-z|^{\gamma}}-1\right), \gamma=\Lambda(n-1) / \lambda-1$ and $\psi(x) \geq \frac{c \delta_{0} \gamma}{4^{\gamma}-1}\left(r_{j}-|x-z|\right)$. In particular, for all points $x$ in $B_{r_{j}}(z) \backslash B_{\frac{r_{j}}{4}}(z)$ belonging to the segment from $z$ to $w$ we have that $\psi(x) \geq \tilde{c}\left(r_{j}-|x-z|\right)=\tilde{c} d\left(x, \partial B_{r_{0}}(y)\right)$, with $\tilde{c}:=\frac{c \delta_{0} \gamma}{4 \gamma-1}$. Letting $z$ vary in $B_{\frac{r_{j}}{4}}\left(y_{j}\right) \cap \partial B_{r_{0}-r_{j}}(y)$, we get (7.28).

For every $x \in B_{\delta_{j}}(0) \cap B_{r_{0}}(y)$, we have

$$
\begin{aligned}
u_{1}-\tilde{m}_{k_{j}} \phi(x) & \geq \tilde{c} d\left(x, \partial B_{r_{0}}(y)\right) \\
& \geq 2 \epsilon \phi(x)
\end{aligned}
$$

for some $\epsilon>0$, hence:

$$
u_{1}-\left(\tilde{m}_{k_{j}}+2 \epsilon\right) \phi(x) \geq 0, \text { for all } x \in B_{\delta_{j}}(0) \cap B_{r_{0}}(y) \text {, for every } j
$$


Let $j_{0}$ be such that $0<\tilde{m}_{\infty}-\tilde{m}_{k_{j}}<\epsilon$ for all $j \geq j_{0}$. Given $j_{0}$, there exists an integer $j_{1} \geq j_{0}$ such that if $j \geq j_{1}$ then $B_{2^{-k_{j}}}(0) \cap B_{r_{0}}(y) \subset B_{\delta_{j_{0}}} \cap B_{r_{0}}(y)$. From (17.29),

$$
u_{1}-\left(\tilde{m}_{k_{j_{0}}}+2 \epsilon\right) \phi(x) \geq 0, \text { for all } x \in B_{2^{-k_{j}}}(0) \cap B_{r_{0}}(y), \quad j \geq j_{1} .
$$

Thus, for $j \geq j_{1}$ we have

$$
\begin{aligned}
u_{1}-\left(\tilde{m}_{k_{j}}+\epsilon\right) \phi(x) & =u_{1}-\left(\tilde{m}_{k_{j_{0}}}+\epsilon\right) \phi(x)-\left(\tilde{m}_{k_{j}}-\tilde{m}_{k_{j_{0}}}\right) \phi(x) \\
& \geq u_{1}-\left(\tilde{m}_{k_{j_{0}}}+\epsilon\right) \phi(x)-\epsilon \phi(x) \\
& \geq u_{1}-\left(\tilde{m}_{k_{j_{0}}}+2 \epsilon\right) \phi(x) \\
& \geq 0, \text { for all } x \in B_{2^{-k_{j}}}(0) \cap B_{r_{0}}(y),
\end{aligned}
$$

which contradicts the definition of $\tilde{m}_{k_{j}}$.

By (7.23) we infer that $\bar{u}=\bar{v}$. Since any subsequence of $\left\{u_{r}\right\}$ converges to the same function, we deduce that the whole sequence $\left\{u_{r}\right\}$ converge, as $r \rightarrow 0$, uniformly in $B_{1}(0)$ to the limit function

$$
\bar{u}=\alpha<x, \nu>^{+}-\beta<x, \nu>^{-}
$$

for some $\alpha, \beta>0$. This means that for any $\epsilon>0$, there exists $\rho>0$ such that for any $0<r<\rho$ and any $x \in \overline{B_{1}(0)}$, then

$$
\left|u_{r}(x)-\alpha<x, \nu>^{+}+\beta<x, \nu>^{-}\right|<\epsilon .
$$

Now, fix $\epsilon>0$ and let $\rho>0$ be defined as above. Fix $z \in B_{\rho}(0)$ and let $r=|z|$. Since $\frac{z}{r} \in \overline{B_{1}(0)}$ we have

that is,

$$
\left|u_{r}\left(\frac{z}{r}\right)-\alpha<\frac{z}{r}, \nu>^{+}+\beta<\frac{z}{r}, \nu>^{-}\right|<\epsilon,
$$

$$
\left|u(z)-\alpha<z, \nu>^{+}+\beta<z, \nu>^{-}\right|<\epsilon r=\epsilon|z|,
$$

which proves (7.22).

Notice that in Lemma 7.3 we did not use that $u$ is a viscosity solution of $\mathcal{F}^{-}(u) \leq 0 \leq \mathcal{F}^{+}(u)$ in $\Omega$. In the next theorem we show that if $u$ satisfies in the viscosity sense these two differential inequalities and has the asymptotic linear behavior (7.22), then we must have $\alpha=\beta$.

Theorem 7.4. Let $u$ be a Lipschitz solution of problem (1.4). Let $0 \in \Gamma$. Assume that exist $\alpha, \beta>0$ and a unit vector $\nu$ such that

$$
u(x)=\alpha<x, \nu>^{+}-\beta<x, \nu>^{-}+o(|x|) .
$$

Then $\alpha=\beta$.

Proof. We first prove that $\beta \geq \alpha$. We argue by contradiction, assuming that $\beta<\alpha$. Fix $h>0$ and let $y:=h \nu$. Notice that $|y|=h$ and $\nu$ is the interior normal unit vector to $B_{h}(y)$ at 0 . Consider the function

$$
\phi(x)=c\left(\frac{h^{\gamma}}{|x-y|^{\gamma}}-1\right), \quad x \neq y
$$


with $\gamma=\frac{\Lambda(n-1)-\lambda}{\lambda}$ and $c>0$. Then, by Lemma 9.1 ,

$$
\begin{cases}\phi(x)>0 & \text { if }|x-y|<h \\ \phi(x)<0 & \text { if }|x-y|>h \\ \frac{\partial}{\partial \nu} \phi(x)=\frac{c \gamma}{h} & \text { if }|x-y|=h \\ \mathcal{M}^{-}(\phi)(x)=0 & \text { if } x \neq y .\end{cases}
$$

Since $\beta<\alpha$, there exists $\epsilon>0$ such that $\beta+\epsilon<\alpha-\epsilon$. Then, we choose $c>0$ such that

$$
\beta+\epsilon<\frac{\partial}{\partial \nu} \phi_{\left.\right|_{\partial B_{h}(y)}}=\frac{c \gamma}{h}<\alpha-\epsilon .
$$

We want to prove that with this choice of $c, \phi \leq u$ in a neighborhood of 0 , for $h$ small enough. In order to prove it, we first show that

$$
\phi \leq u \quad \text { on } \partial B_{(1-s) h}(y)
$$

for $h$ and $s$ small enough.

Observe that the point $w_{1}=s h \nu$ belongs to $\partial B_{(1-s) h}(y)$. Moreover, for any $x \in \partial B_{(1-s) h}(y)$, we have that

$$
<x, \nu>\geq<w_{1}, \nu>=s h
$$

from which we get

$$
u(x)=\alpha<x, \nu>^{+}-\beta<x, \nu>^{-}+o(|x|)=\alpha<x, \nu>^{+}+o(|x|) \geq \alpha s h+o(h) .
$$

Now, let us compute $\phi$ on $\partial B_{(1-s) h}(y)$. If $|x-y|=(1-s) h$ and $c$ satisfies (7.32), then

$$
\begin{aligned}
\phi(x)=c\left(\frac{1}{(1-s)^{\gamma}}-1\right) & =\frac{c}{(1-s)^{\gamma}}\left(1-(1-s)^{\gamma}\right) \\
& =\frac{c}{(1-s)^{\gamma}}(\gamma s+o(s)) \\
& \leq \frac{(\alpha-\epsilon) s h}{(1-s)^{\gamma}}+o(s) h .
\end{aligned}
$$

Let $s>0$ be so small that

$$
\frac{(\alpha-\epsilon) s}{(1-s)^{\gamma}}+o(s)<\left(\alpha-\frac{\epsilon}{2}\right) s .
$$

For such $s$, let $h$ be so small that

$$
\alpha s h+o(h)>\left(\alpha-\frac{\epsilon}{2}\right) s h .
$$

Then, comparing (7.33) with (7.34), we see that $\phi(x)<u(x)$, for any $x \in \partial B_{(1-s) h}(y)$.

Next, let us prove that $\phi>u$ on $\partial B_{h(1+s)}(y)$, for suitable small $h$ and $s$. Let $w_{2}=-s h \nu$, then $w_{2}$ belongs to $\partial B_{h(1+s)}(y)$. Moreover, if $x \in \partial B_{h(1+s)}(y)$, then

$$
<x, \nu>\geq<w_{2}, \nu>=-s h .
$$

Therefore, if $x \in \partial B_{h(1+s)}(y)$, and $<x, \nu>\geq 0$, then

$$
u(x)=\alpha<x, \nu>^{+}+o(|x|) \geq o(h),
$$

and if $\langle x, \nu\rangle \leq 0$, then

$$
u(x)=-\beta<x, \nu>^{-}+o(|x|) \geq-\beta s h+o(h) .
$$


Let us now compute the value of $\phi$ on $\partial B_{h(1+s)}(y)$. If $|x-y|=h(1+s)$, and $c$ satisfies (7.32), then

$$
\phi(x)=-\frac{c}{(1+s)^{\gamma}}\left((1+s)^{\gamma}-1\right)=-\frac{c}{(1+s)^{\gamma}}(\gamma s+o(s)) \leq-\frac{(\beta+\epsilon) s h}{(1+s)^{\gamma}}+o(s) h .
$$

Let $s$ be so small that

$$
-\frac{(\beta+\epsilon) s}{(1+s)^{\gamma}}+o(s) \leq-\left(\beta+\frac{\epsilon}{2}\right) s .
$$

For such $s$, let $h$ be so small that

$$
-\beta s h+o(h) \geq-\left(\beta+\frac{\epsilon}{2}\right) s h .
$$

Then, by (7.35), (7.36) and (7.37), $\phi<u$ on $\partial B_{h(1+s)}(y)$. Putting all together, we have proven that there exist $s, h>0$ such that

$$
\phi<u \quad \text { on } \partial\left(B_{h(1+s)}(y) \backslash B_{h(1-s)}(y)\right) .
$$

Since, in addition

$$
\mathcal{M}^{-}(\phi)=0 \geq \mathcal{F}^{-}(u) \geq \mathcal{M}^{-}(u) \quad \text { on } B_{h(1+s)}(y) \backslash B_{h(1-s)}(y),
$$

the comparison principle combined with the strong maximum principle implies

$$
\phi<u \quad \text { in } B_{h(1+s)}(y) \backslash B_{h(1-s)}(y),
$$

which gives a contradiction at $x=0$.

We conclude that we must have $\alpha \leq \beta$. Arguing similarly as before and using that $\mathcal{F}^{+}(u) \geq 0$ in $\Omega$, one can prove that $\alpha \geq \beta$ and this concludes the proof of the theorem.

7.1. Proof of Theorem 3.2. Theorem 3.2 is a corollary of Lemma 7.3 and Theorem 7.4 ,

\section{Proof of Theorem 3.3}

Consider the following two phase free boundary problem:

$$
\begin{cases}\mathcal{F}^{-}(u)=0 & \text { in } \Omega\left(u^{+}\right) \\ \mathcal{F}^{+}(u)=0 & \text { in } \Omega\left(u^{-}\right) \\ \frac{\partial u^{+}}{\partial \nu_{+}}=\frac{\partial u^{-}}{\partial \nu_{-}} & \text {on } \partial \Omega\left(u^{+}\right),\end{cases}
$$

where $\nu_{ \pm}$is the inner normal vector to $\Omega\left(u^{ \pm}\right)=\left\{u^{ \pm}>0\right\}$.

By Theorem 3.2 we know that any Lipschitz solution to (1.4) satisfies in the viscosity sense (8.1) in $\Omega$. Let us recall the definition of viscosity solution of the problem (8.1) in a given domain $D \subset \mathbb{R}^{n}$, see 3 ] for more details.

Definition 8.1. Let $u$ be a continuous function in $D$. We say that $u$ is a viscosity solution of the problem (8.1) in $D$, if the following holds.

i) $u$ satisfies in the viscosity sense

$$
\begin{cases}\mathcal{F}^{-}(u)=0 & \text { in }\{u>0\} \cap D \\ \mathcal{F}^{+}(u)=0 & \text { in }\{u<0\} \cap D .\end{cases}
$$

ii) If there exists a tangent ball at $x_{0} \in \partial\{u>0\} \cap D, B$, such that either $B \subset\{u>0\} \cap D$ or $B \subset\{u<0\} \cap D$, then

$$
u(x)=\alpha<x-x_{0}, \nu_{+}>+o\left(\left|x-x_{0}\right|\right)
$$

with $\alpha>0$ and $\nu_{+}$the normal vector to $\partial B$ at $x_{0}$ pointing inward to $\{u>0\} \cap D$. 
In this section we prove that for any viscosity solution to the free boundary problem (8.1) the following holds: if the free boundary is flat around 0 , meaning that it can be trapped in a small neighborhood of the graph of a Lipschitz function, then in a neighborhood of 0 , it is a $C^{1, \alpha}$ surface. Theorem 3.3 will follow as a corollary of this result. To prove that flatness implies $C^{1, \alpha}$, we follow the classical sup-convolution method developed by Caffarelli in the papers [5, 6] for the Laplace operator and extended by Wang [20, 21 to fully-nonlinear elliptic operators. Problem (8.1) differs from the one studied in [20,21] since $u$ satisfies two different equations in $\Omega\left(u^{+}\right)$and $\Omega\left(u^{-}\right)$. However the regularity theory developed in those papers can be extended to our problem and some simplifications arise due to the specific free boundary condition here considered: $\frac{\partial u^{+}}{\partial \nu_{+}}=\frac{\partial u^{-}}{\partial \nu_{-}}$.

Following the classical theory, we first prove that Lipschitz free boundaries are $C^{1, \alpha}$ and then we prove that flat free boundaries are Lipschitz.

8.1. Lipschitz free boundaries are $C^{1, \alpha}$. For $r>0$, let $\mathcal{C}_{r}$ be the cylinder defined as $\mathcal{C}_{r}:=$ $B_{r}^{\prime}(0) \times(-r, r)$, where $B_{r}^{\prime}(0)$ is the ball centered at 0 of radius $r$ of $\mathbb{R}^{n-1}$.

Proposition 8.2. Let $u$ be a viscosity solution of the problem (8.1) in $\mathcal{C}_{1}=B_{1}^{\prime}(0) \times(-1,1)$. Assume that $0 \in \partial \Omega\left(u^{+}\right)$and that

$$
\mathcal{C}_{1} \cap \Omega\left(u^{+}\right)=\left\{\left(x^{\prime}, x_{n}\right) \mid x_{n}>g\left(x^{\prime}\right)\right\}
$$

where $g$ is a Lipschitz continuous function. Then in $B_{\frac{1}{2}}^{\prime}(0), g$ is a $C^{1, \alpha}$-function, for some $0<\alpha \leq 1$.

Proof. The proof of the proposition follows by [5] (see also [3]) and [20. As already pointed out, even though we have different operators on each side of the free boundary, the classical regularity theory still applies. For completion of this paper, we will sketch the main parts of the method highlighting the parts that are simplified in our problem due to the free boundary condition in (8.1).

Step 1: Existence of a cone of monotonicity.

By [20, Lemma 2.5] applied to $u^{+}$and the operator $\mathcal{F}^{-}$, there exists $\delta>0$ such that $\partial_{x_{n}} u^{+} \geq 0$ in the set $\mathcal{C}_{\delta} \cap\left\{x_{n}>g\left(x^{\prime}\right)\right\}$. Also, applying the same Lemma to $u^{-}$and the operator $F(u)=$ $-\mathcal{F}^{+}(-u)$, we have that $\partial_{-x_{n}} u^{-}=-\partial_{x_{n}} u^{-} \geq 0$ on the set $\mathcal{C}_{\delta} \cap\left\{x_{n}<g\left(x^{\prime}\right)\right\}$. Thus, since $u=u^{+}-u^{-}$, we conclude that $u$ is monotone increasing in the direction of $e_{n}=(0, \ldots, 0,1)$ in $\mathcal{C}_{\delta}$. The same is true for any direction $\tau$ in the cone determined by $L$, the Lipschitz constant of $g$; that is, let $\Gamma\left(\theta, e_{n}\right)$ be the cone with axis $e_{n}$ and semi-opening $\theta$ given by $\operatorname{cotan} \theta=L$, then $u$ is monotone increasing in the direction of $\tau \in \Gamma\left(\theta, e_{n}\right)$, in $\mathcal{C}_{\delta} . \Gamma\left(\theta, e_{n}\right)$ is called the monotonicity cone of $u$.

Step 2: Improvement of the Lipschitz regularity away from the free boundary.

We may suppose that the monotonicity cone exists for all points $x \in \mathcal{C}_{1}$, by using, if necessary, the invariance by elliptic dilation of the problem. The monotonicity of $u$ along the directions of $\Gamma\left(\theta, e_{n}\right)$ implies that for every small $\tau \in \Gamma\left(\frac{\theta}{2}, e_{n}\right)$,

$$
\sup _{z \in B_{\epsilon}(x)} u(z-\tau) \leq u(x)
$$

for every $x \in \mathcal{C}_{1-\epsilon}$, where $\epsilon=|\tau| \sin \left(\frac{\theta}{2}\right)$. Let $x_{0}:=\frac{3}{4} e_{n} \in \mathcal{C}_{1}$. The proof of Lemma 4.6 of [3] which uses Harnack inequality and Schauder estimates, can be adapted to our case to improve the opening of the monotonicity cone in a neighborhood of $x_{0}$. The result goes as follows: there 
exist positive constants $b$ and $c$ such that for every small $\tau \in \Gamma\left(\frac{\theta}{2}, e_{n}\right)$ and every $x \in B_{\frac{1}{8}}\left(x_{0}\right)$

$$
\sup _{z \in B_{(1+b) \epsilon}(x)} u(z-\tau) \leq u(x)-c \epsilon u\left(x_{0}\right),
$$

with $\epsilon=|\tau| \sin \left(\frac{\theta}{2}\right)$.

Step 3: Construction of a family of subsolutions of variable radii.

Here the main technique is the sup-convolution method to construct a family of subsolutions of the form

$$
w_{\varphi}(x)=\sup _{z \in B_{\varphi(x)}(x)} u(z-\tau),
$$

for small $\tau \in \Gamma\left(\frac{\theta}{2}, e_{n}\right)$, to compare with the solution $u$ of (8.1). In order to apply the comparison principle, it is necessary to study the properties of the sup-convolution function and since problem (8.1) is invariant by translations, it is enough to do it before translations, that is with $u(\cdot-\tau)$ replaced by $u$.

For $0<r \leq \frac{1}{8}, 0<h<1$, there exists a family of functions $\varphi_{t}, 0 \leq t \leq 1$, with $\varphi_{t} \in$ $C^{2}\left(\overline{B_{1}(0)} \backslash B_{\frac{r}{2}}\left(x_{0}\right)\right), x_{0}=\frac{3}{4} e_{n}$, with the following properties:

(a) $1 \leq \varphi_{t} \leq 1+t h$

(b) $\varphi_{t} \equiv 1$ outside $B_{\frac{7}{8}}(0)$,

(c) $\varphi_{t} \geq 1+\lambda t h$, in $B_{\frac{1}{2}}(0)$, for some $\lambda=\lambda(r)$,

(d) $\left|\nabla \varphi_{t}\right| \leq C t h$.

Moreover, if we define

(e) then

$$
v_{\varphi_{t}}(x):=\sup _{z \in B_{\varphi_{t}(x)}(x)} u(z)
$$

$$
\begin{array}{ll}
\mathcal{F}^{-}\left(v_{\varphi_{t}}\right) \geq 0 & \text { in } \Omega\left(v_{\varphi_{t}}^{+}\right), \\
\mathcal{F}^{+}\left(v_{\varphi_{t}}\right) \geq 0 & \text { in } \Omega\left(v_{\varphi_{t}}^{-}\right),
\end{array}
$$

and if $\left|\nabla \varphi_{t}\right|<1$ then

(f) for every point of $\partial \Omega\left(v_{\varphi_{t}}^{+}\right)$there is a tangent ball contained in $\Omega\left(v_{\varphi_{t}}^{+}\right)$,

(g) for every point $x_{1} \in \partial \Omega\left(v_{\varphi_{t}}^{+}\right)$, there exists $\bar{\alpha}$ such that

$$
v_{\varphi_{t}}(x) \geq \bar{\alpha}<x-x_{1}, \bar{\nu}>+o\left(\left|x-x_{1}\right|\right),
$$

where $\bar{\nu}$ is the normal vector of $\partial \Omega\left(v_{\varphi_{t}}^{+}\right)$pointing inward $\Omega\left(v_{\varphi_{t}}^{+}\right)$.

Properties (a)-(e) are proven in [20, Lemmas 3.4, 3.5]. Since in [20] only concave operators (like $\mathcal{F}^{-}$) are considered, for the second inequality in (e) we refer to [11, Proposition 1.1] where more general operators, not necessary concave, are taken into account. Property (f) is proven in [3, Lemma 4.9]. Let us prove (g). Note that $u \leq v_{\varphi_{t}}$, therefore $\Omega\left(u^{+}\right) \subset \Omega\left(v_{\varphi_{t}}^{+}\right)$. Now, let $x_{1} \in \partial \Omega\left(v_{\varphi_{t}}^{+}\right)$, then there exists $y_{1} \in \partial \Omega\left(u^{+}\right)$such that $v_{\varphi_{t}}\left(x_{1}\right)=u\left(y_{1}\right)=0$. Note that we must have $y_{1} \in \partial B_{\varphi_{t}\left(x_{1}\right)}\left(x_{1}\right)$. Thus, $B_{\varphi_{t}\left(x_{1}\right)}\left(x_{1}\right)$ is tangent to $\partial \Omega\left(u^{+}\right)$at $y_{1}$ contained in $\Omega\left(u^{-}\right)$and according to Definition 8.1 we have that

$$
u(y)=\alpha<y-y_{1}, \nu>+o\left(\left|y-y_{1}\right|\right),
$$


where $\nu$ is the unit normal vector to $\partial \Omega\left(u^{+}\right)$at $y_{1}$ pointing inward $\Omega\left(u^{+}\right)$. If $y=x+\varphi_{t}(x) \nu$, since $y_{1}=x_{1}+\varphi_{t}\left(x_{1}\right) \nu$, we obtain the asymptotic behavior of $v_{\varphi_{t}}$ in a neighborhood of $x_{1}$ :

$$
\begin{aligned}
v_{\varphi_{t}}(x) & \geq u(y) \\
& =\alpha<x+\varphi_{t}(x) \nu-x_{1}-\varphi_{t}\left(x_{1}\right) \nu, \nu>+o\left(\left|x-x_{1}\right|\right) \\
& =\alpha<x-x_{1}+\left(\varphi_{t}(x)-\varphi_{t}\left(x_{1}\right)\right) \nu, \nu>+o\left(\left|x-x_{1}\right|\right) .
\end{aligned}
$$

We replace $\varphi_{t}(x)-\varphi_{t}\left(x_{1}\right)$ by $\left\langle x-x_{1}, \nabla \varphi_{t}\left(x_{1}\right)>+o\left(\left|x-x_{1}\right|\right)\right.$ in the previous inequality and simplify to obtain

Thus, if we let

$$
v_{\varphi_{t}}(x) \geq \alpha<x-x_{1}, \nu+\nabla \varphi_{t}\left(x_{1}\right)>+o\left(\left|x-x_{1}\right|\right)
$$

$$
\bar{\alpha}:=\alpha\left|\nu+\nabla \varphi_{t}\left(x_{1}\right)\right|, \quad \bar{\nu}:=\frac{\nu+\nabla \varphi_{t}\left(x_{1}\right)}{\left|\nu+\nabla \varphi_{t}\left(x_{1}\right)\right|},
$$

we obtain (8.5). By Lemma 4.9 in [3], $\bar{\nu}$ is the unit normal vector to $\partial \Omega^{+}\left(v_{\varphi_{t}}\right)$ at $x_{1}$ pointing inward $\Omega^{+}\left(v_{\varphi_{t}}\right)$. We note that in our problem we do not need the correctors used in the supconvolution method to obtain the correct asymptotic behavior of $v_{\varphi_{t}}$ on points on the free boundary (see [3, Lemma 4.12]).

\section{Step 4: Comparison with subsolutions.}

In what follows, we will have to compare the solution $u$ of (8.1) with the functions

$$
w_{t}(x):=\sup _{z \in B_{\epsilon \varphi_{b t}(x)}(x)} u(z-\tau), \quad x \in D,
$$

for small $\tau \in \Gamma\left(\frac{\theta}{2}, e_{n}\right)$, where $D:=B_{\frac{9}{10}}(0) \backslash B_{\frac{1}{8}}\left(x_{0}\right), b$ is defined in (8.3), $\epsilon=|\tau| \sin \left(\frac{\theta}{2}\right)$ and $\varphi_{t}$ is the family of functions defined in Step 3. By (d) in Step 3 we can choose $h$ small so that $\epsilon\left|\nabla \varphi_{b t}\right|<1$, therefore by (f), we have that

$$
\text { for every point of } \partial \Omega\left(w_{\varphi_{t}}^{+}\right) \text {there is a tangent ball contained in } \Omega\left(w_{\varphi_{t}}^{+}\right) \text {. }
$$

Now, having on hands (8.8) and the asymptotic development (8.5) we can show the following comparison result between $u$ and $w_{t}$ : suppose that

$$
u \geq w_{t} \text { in } D, \quad u>w_{t} \text { in } \Omega\left(w_{t}^{+}\right), \text {then } \partial \Omega\left(w_{t}^{+}\right) \text {and } \partial \Omega\left(w_{t}^{+}\right) \text {cannot touch. }
$$

The proof is given in [3, Lemma 2.1]. We perform it here for reader's convenience. By (8.9), we know that $\Omega\left(w_{t}^{+}\right) \subset \Omega\left(u^{+}\right)$. Suppose by contradiction that there exists $x_{1} \in \partial \Omega\left(w_{t}^{+}\right) \cap$ $\partial \Omega\left(u^{+}\right)$, then, by (8.8), there exists a tangent ball to $\partial \Omega\left(u^{+}\right)$at $x_{1}$ contained in $\Omega\left(u^{+}\right)$. Thus, according to Definition 8.1, we have

$$
u(x)=\alpha<x-x_{1}, \nu>+o\left(\left|x-x_{1}\right|\right) .
$$

and by (8.5), there exists $\eta>0$ such that

$$
w_{t}(x) \geq \eta<x-x_{1}, \nu>+o\left(\left|x-x_{1}\right|\right) .
$$

Note that here $\bar{\nu}=\nu$. Since $w_{t} \leq u$ and $w_{t}\left(x_{1}\right)=u\left(x_{1}\right)=0$, by (8.10) and (8.11), it follows that

$$
\alpha=\eta
$$

We have that $u-w_{t}$ is a supersolution for $\mathcal{F}^{-}$in $\Omega\left(w_{t}^{+}\right)$, since by (c) in Proposition 2.1, (8.1) and (e), in $\Omega\left(w_{t}^{+}\right) \subset \Omega\left(u^{+}\right)$we have

$$
0=\mathcal{F}^{-}(u) \geq \mathcal{F}^{-}\left(u-w_{t}\right)+\mathcal{F}^{-}\left(w_{t}\right) \geq \mathcal{F}^{-}\left(u-w_{t}\right) .
$$


Since $u>w_{t}$ in $\Omega\left(w_{t}\right)$, by the Hopf principle there exists $\delta>0$ such that

$$
\left(u-w_{t}\right)\left(x_{1}+h \nu\right) \geq \delta h,
$$

for all small $h>0$. This is a contradiction, since by (8.10), (8.11) and (8.12), we have that

$$
\left(u-w_{t}\right)\left(x_{1}+h \nu\right) \leq o(h) .
$$

Thus, we conclude that $\partial \Omega\left(w_{t}^{+}\right)$and $\partial \Omega\left(u^{+}\right)$cannot touch.

Step 5: Carrying the improvement of Step 2 to the free boundary.

The improvement obtained in Step 2 needs to be carried to the free boundary, in $B_{1 / 2}(0)$, giving up a little bit of the interior improvement.

In order to do this, we consider the family of functions $w_{t}$ defined in (8.7). Let $D:=$ $B_{\frac{9}{10}}(0) \backslash B_{\frac{1}{8}}\left(x_{0}\right)$, let us check that the following conditions are satisfied:

i) $w_{0} \leq u$ in $D$,

ii) $w_{t} \leq u$ on $\partial D$ and $w_{t}<u$ in $\overline{\Omega\left(w_{t}^{+}\right)} \cap \partial D$,

iii) the family $\Omega\left(w_{t}^{+}\right)$is uniformly continuous, that is, for every $\epsilon>0$,

$$
\Omega\left(w_{t_{1}}^{+}\right) \subset \mathcal{N}_{\epsilon}\left(\Omega\left(w_{t_{2}}^{+}\right)\right)
$$

whenever $\left|t_{1}-t_{2}\right|<\delta(\epsilon)$, where $\mathcal{N}_{\epsilon}\left(\Omega\left(w_{t_{2}}^{+}\right)\right)$is a $\epsilon$-neighborhood of $\Omega\left(w_{t_{2}}^{+}\right)$.

By (a) in Step 3, $\varphi_{0} \equiv 1$ and thus by (8.2), if $x \in D$, we have

$$
w_{0}(x)=\sup _{z \in B_{\epsilon}(x)} u(z-\tau) \leq u(x),
$$

which is (i).

By (b) in Step 3, and (8.2) if $x \in \partial B_{\frac{9}{10}}(0)$, then

$$
w_{t}(x)=\sup _{z \in B_{\epsilon}(x)} u(z-\tau) \leq u(x)
$$

and the inequality is strict in $\overline{\Omega\left(w_{t}^{+}\right)}$, by taking any $\epsilon^{\prime}<\epsilon$ if necessary. If $x \in \partial B_{\frac{1}{8}}\left(x_{0}\right)$ by (a) of Step 3 and (8.3), we have that (since $t, h \leq 1$ ),

$$
w_{t}(x) \leq \sup _{z \in B_{(1+t b h) \epsilon}(x)} u(z-\tau) \leq \sup _{z \in B_{(1+b) \epsilon}(x)} u(z-\tau)<u(x) .
$$

Combining (8.14) and (8.15) yields (ii).

Finally, (iii) follows from the definition of the functions $w_{t}$, (8.7).

Now, from (i)-(iii) and by using (8.9), we can conclude that

$$
w_{t} \leq u \text { in } D \text { for every } t \in[0,1] \text {. }
$$

The proof of (8.16) is given in [3, Theorem 2.2] in the case of the Laplace operator and we present it here for the sake of completeness. For that, let $E:=\left\{t \in[0,1] \mid v_{t} \leq u\right.$ in $\left.\bar{D}\right\}$. By (i) $0 \in E$. $E$ is obviously closed. Let us show that it is open. If $t_{0} \in E$, that is $v_{t_{0}} \leq u$ in $D$, from (ii) and the strong maximum principle it follows that $v_{t_{0}}<u$ in $\Omega\left(v_{t_{0}}^{+}\right) \cap D$. By (ii) and (8.9) we have that $\overline{\Omega\left(v_{t_{0}}\right)} \cap D$ is compactly supported in $\Omega\left(u^{+}\right) \cap D$ up to the boundary of $D$. From (iii), there exists $\delta>0$ such that $\overline{\Omega\left(v_{t}\right)} \cap D$ is compactly supported in $\Omega\left(u^{+}\right) \cap D$ for all $t$ such that $\left|t-t_{0}\right|<\delta$. Thus, for such values of $t$, by (ii) and (e) of Claim 1 we have

$$
\begin{gathered}
\mathcal{F}^{-}\left(v_{\varphi_{t}}\right) \geq 0=\mathcal{F}^{-}(u) \quad \text { in } \Omega\left(v_{\varphi_{t}}^{+}\right) \cap D, \\
v_{\varphi_{t}} \leq u \quad \text { on } \partial\left(\Omega\left(v_{\varphi_{t}}^{+}\right) \cap D\right)
\end{gathered}
$$


and by the comparison principle, $v_{\varphi_{t}} \leq u$ in $\Omega\left(v_{\varphi_{t}}^{+}\right) \cap D$. Similarly, since

$$
\mathcal{F}^{+}\left(v_{\varphi_{t}}\right) \geq 0=\mathcal{F}^{+}(u) \text { in } \Omega\left(u^{-}\right) \cap D,
$$

and

$$
v_{\varphi_{t}} \leq u \quad \text { on } \partial\left(\Omega\left(u^{-}\right) \cap D\right),
$$

we have that $v_{\varphi_{t}} \leq u$ in $\Omega\left(u^{-}\right) \cap D$. Clearly $v_{\varphi_{t}} \leq 0 \leq u$ in $\overline{\Omega\left(u^{+}\right)} \cap \overline{\Omega\left(v_{\varphi_{t}}^{-}\right)} \cap D$. We conclude that $v_{\varphi_{t}} \leq u$ in $D$ and the openness of $E$ follows. Since $E$ is both an open and closed nonempty subset of $[0,1]$, we must have $E=[0,1]$. This proves (8.16).

Inequality (8.16) holds in particular for $t=1$ and hence using (c) in Step 3 we obtain that, on $B_{1 / 2}(0)$,

$$
\begin{aligned}
u & \geq w_{1} \\
& =\sup _{z \in B_{\epsilon \varphi_{b}(x)}(x)} u(z-\tau) \\
& \geq \sup _{z \in B_{\epsilon(1+(\lambda h) b)}(x)} u(z-\tau),
\end{aligned}
$$

which implies the desired improvement of the cone of monotonicity across the free boundary. The original radius $\epsilon$ in (8.13) was first improved to $\epsilon+\epsilon b$ far from the free boundary (see (8.3) $)$, and at the free boundary the radius became $\epsilon+(\lambda h) \epsilon b$. Since $\lambda h<1$, a little bit of opening in the cone has to be given up in order to bring the improvement across the free boundary (see Theorem 4.2 and Lemma 4.4 in [3] for details).

\section{Step 6: Basic iteration.}

Rescaling and repeating Steps 2-5 we obtain that the free boundary is $C^{1, \alpha}$ in $\mathcal{C}_{\frac{1}{2}}$, see the proof of Theorem 4.1 in [3] for details.

8.2. Flat free boundaries are Lipschitz. In this subsection we prove that if $u$ is a solution of the free boundary problem (8.1) and the free boundary can be trapped in a narrow neighborhood in between two Lipschitz graphs, then the free boundary is actually Lipschitz. Let us recall the definition of $\epsilon$-monotone function.

Definition 8.3. We say that $u$ is $\epsilon$-monotone in the cylinder $\mathcal{C}_{1}$ along a direction $\tau$, with $|\tau|=1$, if for all $x \in \mathcal{C}_{1}$,

$$
u(x+l \tau) \geq u(x),
$$

for all $l \geq \epsilon$ such that $x+l \tau \in \mathcal{C}_{1}$.

The $\epsilon$-monotonicity can be reformulated equivalently as follows, see [3].

Definition 8.4. We say that $u$ is $\epsilon_{0}$-monotone in the cylinder $\mathcal{C}_{1}$ along the directions of the cone $\Gamma(\theta, e)$ if for all $x \in \mathcal{C}_{1}$,

$$
\sup _{y \in B_{\epsilon \sin \theta}(x)} u(y-\epsilon e) \leq u(x),
$$

for any $\epsilon \geq \epsilon_{0}$ such that $B_{\epsilon \sin \theta}(x-\epsilon e) \subset \mathcal{C}_{1}$.

As in Subsection 8, in the definition above $\Gamma(\theta, e)$ denotes the cone of semi-opening $\theta$ and axis $e$.

Remark 8.5. If $u$ is $\epsilon$-monotone in $\mathcal{C}_{1}$ according to Definition 8.4, then the level surfaces of $u$ in $\mathcal{C}_{1}, \partial\{u>t\}$, are contained in a $(1-\sin \theta) \epsilon$ size of the graph of a Lipschitz function $g$ with Lipschitz constant $L=\operatorname{cotan} \theta<1$, see [3]. 
Proposition 8.6. Let $\frac{\pi}{4}<\theta<\frac{\pi}{2}$ and let $u$ be a viscosity solution of the problem (8.1) in $\mathcal{C}_{1}=B_{1}^{\prime} \times(-1,1)$. Assume that $0 \in \partial \Omega\left(u^{+}\right)$. Then there exists $\epsilon=\epsilon(\theta)$ such that if $u$ is $\epsilon$-monotone in $\mathcal{C}_{1-\epsilon}=B_{1-\epsilon}^{\prime} \times(-1+\epsilon, 1-\epsilon)$ along any direction $\tau$ in the cone $\Gamma(\theta, e)$, then $u$ is fully monotone in $\mathcal{C}_{\frac{1}{2}}=B_{\frac{1}{2}}^{\prime} \times\left(-\frac{1}{2}, \frac{1}{2}\right)$ along any direction $\tau \in \Gamma\left(\theta_{1}, e\right)$ with $\theta_{1}=\theta_{1}(\theta, \epsilon)$.

Proof. The proof of this result follows from [5] (see also [3]) and [20]. We will sketch the proof below.

Step 1: Full monotonicity of $u$ outside a strip of size $M \epsilon$ of the free boundary.

By Lemma 1 in [21] there exists $M>1$ such that in $\mathcal{C}_{1} \backslash \mathcal{N}_{M \epsilon}$, where

$$
\mathcal{N}_{M \epsilon}:=\left\{x \in \mathcal{C}_{1} \mid d\left(x, \partial \Omega\left(u^{+}\right)\right)<M \epsilon\right\}
$$

$u$ is actually fully monotone along any direction of $\tau \in \Gamma(\theta, e)$.

Step 2: Construction of a family of subsolutions of variable radii.

Following the method developed in [6], we need to construct a family of subsolutions of the form

$$
w(x)=\sup _{z \in B_{\varphi(x)}(x)} u(z-\lambda \epsilon e),
$$

for some $\lambda \in(0,1)$, to compare with the solution $u$ of (8.1). Up to a change of coordinates, we can assume that

$$
e=e_{n}
$$

Since $u$ is $\epsilon$-monotone, by Remark 8.5 there exists $g: \mathbb{R}^{n-1} \rightarrow \mathbb{R}$ with $g(0)=0$ and Lipschitz constant $L=\operatorname{cotan} \theta<1$, such that if

$$
A:=\left\{\left(x^{\prime}, x_{n}\right) \in \mathbb{R}^{n} \mid x_{n}=g\left(x^{\prime}\right)\right\},
$$

then

$$
\partial \Omega\left(u^{+}\right) \subset \mathcal{N}_{\epsilon}(A)
$$

where

$$
\mathcal{N}_{\epsilon}(A):=\left\{x \in \mathcal{C}_{1} \mid d(x, A)<\epsilon\right\} .
$$

By Lemmas 2 and 3 in [21] and Proposition 1.1 in [11, for any given $\delta>0$, there exists a family of $C^{2}$-functions, $\varphi_{t}, 0 \leq t \leq 1$, defined on $\mathcal{C}:=\overline{B_{1}^{\prime}(0)} \times[-2 L, 2 L]$, with the following properties:

a) $1 \leq \varphi_{t} \leq 1+t$,

b) $\varphi_{t} \equiv 1$ on $A_{\delta}:=\{x \in \mathcal{C} \mid d(x, A \cap \partial \mathcal{C})<\delta\}$,

c) in the set $\{x \in \mathcal{C} \mid d(x, \partial \mathcal{C})>\delta\}$,

$$
\varphi_{t} \geq 1+t\left(1-\frac{C \delta}{d(x, \partial \mathcal{C})^{2}}\right)
$$

d) $\left|\nabla \varphi_{t}\right| \leq \frac{C t}{\delta}$.

Moreover,

e) if we define

$$
v_{\varphi_{t}}(x):=\sup _{z \in B_{\varphi_{t}(x)}(x)} u(z),
$$

then $v_{\varphi_{t}}$ satisfies

$$
\begin{array}{ll}
\mathcal{F}^{-}\left(v_{\varphi_{t}}\right) \geq 0 & \text { in } \Omega\left(v_{\varphi_{t}}^{+}\right), \\
\mathcal{F}^{+}\left(v_{\varphi_{t}}\right) \geq 0 & \text { in } \Omega\left(v_{\varphi_{t}}^{-}\right),
\end{array}
$$

and if $\left|\nabla \varphi_{t}\right|<1$ then 
f) for every point of $\partial \Omega^{+}\left(v_{\varphi_{t}}\right)$ there is a tangent ball contained in $\Omega^{+}\left(v_{\varphi_{t}}\right)$,

g) if

$$
0<\sin \bar{\theta} \leq \frac{1}{1+\left|\nabla \varphi_{t}\right|}\left(\sin \theta-\frac{\epsilon}{2 \varphi_{t}} \cos ^{2} \theta-\left|\nabla \varphi_{t}\right|\right)
$$

then $v_{\varphi_{t}}$ is monotone in the cone $\Gamma\left(\bar{\theta}, e_{n}\right)$; in particular its level surfaces are Lipschitz graphs, in the direction of $e_{n}$, with Lipschitz constant $\bar{L} \leq \operatorname{cotan} \bar{\theta}$.

Finally, as in the proof of Proposition 8.2, if $\left|\nabla \varphi_{t}\right|<1$, the function $v_{\varphi_{t}}$ has the following behavior at points of $\partial \Omega\left(v_{\varphi_{t}}^{+}\right)$

h) for every point $x_{1} \in \partial \Omega\left(v_{\varphi_{t}}^{+}\right)$there exists $\bar{\alpha}>0$ such that

$$
v_{\varphi_{t}}(x) \geq \bar{\alpha}<x-x_{1}, \bar{\nu}>+o\left(\left|x-x_{1}\right|\right),
$$

where $\bar{\nu}$ is the normal vector of $\partial \Omega\left(v_{\varphi_{t}}^{+}\right)$pointing inward $\Omega\left(v_{\varphi_{t}}^{+}\right)$.

Step 3: Comparison with subsolutions. In what follows, we will have to compare the solution $u$ of (8.1) with the functions

$$
w_{t}(x):=\sup _{z \in B_{\sigma \varphi_{t}(x)}(x)} u\left(z-\lambda \epsilon e_{n}\right),
$$

for $\sigma, \lambda \in(0,1)$ to be determined, where $\varphi_{t}$ is the family of functions defined in Step 2. We first notice that from the $\epsilon$-monotonicity of $u$ (Definition 8.4), for $1-\lambda<\sqrt{2} / 2$, we have

$$
\sup _{z \in B_{\epsilon(\sin \theta-(1-\lambda))}(x)} u\left(z-\lambda \epsilon e_{n}\right) \leq \sup _{z \in B_{\epsilon \sin \theta}(x)} u\left(z-\epsilon e_{n}\right) \leq u(x),
$$

since $B_{\epsilon(\sin \theta-(1-\lambda))}\left(x-\lambda \epsilon e_{n}\right) \subset B_{\epsilon \sin \theta}\left(x-\epsilon e_{n}\right)$.

For any $\eta>0$ and $A$ defined as in (8.17), let us denote by $\mathcal{N}_{\eta}(A)$ the $\eta$-neighborhood of $A$, defined by

$$
\mathcal{N}_{\eta}(A):=\{x \in \mathcal{C} \mid d(x, A)<\eta\} .
$$

By Step 1 and (8.18), $u$ is fully monotone in the directions of $\Gamma\left(\theta, e_{n}\right)$, outside the set $\mathcal{N}_{2 M \epsilon}(A)$. Therefore,

$$
\sup _{z \in B_{\lambda \epsilon \sin \theta}(x)} u\left(z-\lambda \epsilon e_{n}\right) \leq u(x) \quad \text { for } x \notin \mathcal{N}_{2 M \epsilon}(A)
$$

We now choose

$$
\sigma:=\epsilon(\sin \theta-(1-\lambda)), \quad \lambda \geq \frac{3}{2}-\frac{\sqrt{2}}{2}, \quad \delta:=\epsilon^{\frac{1}{2}} .
$$

Then the family of functions $w_{t}$ in (8.19) is well defined in $\mathcal{C}_{1-\epsilon} \cap \mathcal{N}_{2 M \epsilon}(A)$. Moreover, (e)-(h) of Step 2 hold true for $\epsilon$ (and thus $\sigma$ ) small enough. Since $\sigma$ defined as in (8.22) satisfies $\sigma<\lambda \epsilon \sin \theta$, by (a) of Step 2 we can choose $\bar{t}>0$ so small that

$$
\sigma \varphi_{t} \leq \lambda \epsilon \sin \theta, \quad \text { for } 0 \leq t \leq \bar{t} .
$$

By (e)-(h) of Step 2, the functions $w_{t}, 0 \leq t \leq 1$, satisfy

$$
\mathcal{F}^{-}\left(w_{t}\right) \geq 0 \text { in } \Omega\left(w_{t}^{+}\right),
$$

for any point of $\partial \Omega\left(w_{t}^{+}\right)$there is a tangent ball contained in $\Omega\left(w_{t}^{+}\right)$

For every point $x_{1} \in \partial \Omega\left(w_{t}^{+}\right)$, there exists $\bar{\alpha}>0$ such that

$$
w_{t}(x) \geq \bar{\alpha}<x-x_{1}, \bar{\nu}>+o\left(\left|x-x_{1}\right|\right) \text {. }
$$


Let us show that for all $0 \leq t \leq \bar{t}$,

$$
w_{t}(x) \leq u(x) \text { for } x \in \partial\left(\mathcal{N}_{2 M \epsilon}(A) \cap \mathcal{C}_{1-4 \epsilon}\right) .
$$

If $x \in \partial\left(\mathcal{N}_{2 M \epsilon}(A)\right) \cap \mathcal{C}_{1-4 \epsilon}$, then by (8.23) and (8.21), we have that

$$
w_{t}(x) \leq \sup _{z \in B_{\lambda \epsilon \sin \theta}(x)} u\left(z-\lambda \epsilon e_{n}\right) \leq u(x) .
$$

If $\left.x \in \mathcal{N}_{2 M \epsilon}(A)\right) \cap \partial\left(\mathcal{C}_{1-4 \epsilon}\right)$, then, since for $\epsilon$ small enough $\delta=\epsilon^{1 / 2}>4 \epsilon$, by (b) of Step 2, $\varphi_{t}(x)=1$. Thus, by the definition of $\sigma$ in (8.22) and (8.20), for $\left.x \in \mathcal{N}_{2 M \epsilon}(A)\right) \cap \partial\left(\mathcal{C}_{1-4 \epsilon}\right)$,

$$
w_{t}(x)=\sup _{z \in B_{\epsilon(\sin \theta-(1-\lambda))}(x)} u\left(z-\lambda \epsilon e_{n}\right) \leq u(x) .
$$

This concludes the proof of (8.27).

Finally, by (8.27) and using that the functions $w_{t}$ satisfy (8.24)-(8.26), arguing as in Step 5 of the proof of Proposition 8.2, we infer that, for $0 \leq t \leq \bar{t}$,

$$
w_{t}(x) \leq u(x) \text { for all } x \in \mathcal{N}_{2 M \epsilon}(A) \cap \mathcal{C}_{1-4 \epsilon} .
$$

Step 4: From the $\epsilon$-monotonicity to the $\lambda \epsilon$-monotonicity.

Arguing as in 6] (see also Lemma 5.7 in [3]), by (8.29) and (c) of Step 2, we have that there exists $c_{0}>0$ such that in $\mathcal{N}_{2 M \epsilon}(A) \cap \mathcal{C}_{1-4 \epsilon^{1 / 8}}$

$$
\sup _{\lambda \epsilon \sin \left(\theta-c_{0} \epsilon^{1 / 4}\right)} u\left(z-\lambda \epsilon e_{n}\right) \leq u(x),
$$

that is $u$ is $\lambda \epsilon$-monotone in any direction of the cone of directions $\Gamma\left(\theta-c_{0} \epsilon^{1 / 4}, e_{n}\right)$.

\section{Step 5: Basic iteration.}

Rescaling and repeating Steps 1-4, we obtain that the free boundary is Lipschitz in $\mathcal{C}_{\frac{1}{2}}$, see the proof of Theorem 5.1 in 3 for details.

8.3. Proof of Theorem 3.3. Let $u$ be a solution of (1.4). Then, by Theorem 3.2, $u$ is a solution of the free boundary problem (8.1) in the sense of Definition 8.1. Let $z \in \Gamma$ be a regular point. Assume without loss of generality that $z=0$. By Corollary 7.2 , there exists $r_{j} \rightarrow 0$ as $j \rightarrow+\infty$ with the following property: for any $\epsilon>0$ there exists $J \in \mathbb{N}$ such that for any $j \geq J$, all the level sets of $u_{r_{j}}(x)=u\left(r_{j} x\right) / r_{j}$ in $B_{2}(0)$ are $\epsilon$-flat. Also, by scaling invariance $u_{r_{j}}$ is solution of (8.1) in the cylinder $\mathcal{C}_{1}=B_{1}^{\prime}(0) \times(-1,1)$. We can now apply Propositions 8.2 and 8.6 to conclude that there is $J \in \mathbb{N}$ such that for any $j \geq J$ the set $\partial \Omega\left(\left(u_{1}\right)_{r_{j}}\right) \cap B_{\frac{1}{4}}(0)$ is of class $C^{1, \alpha}$ for some $0<\alpha \leq 1$. Therefore, the same is true for $\Gamma \cap B_{\frac{r_{j}}{4}}(0)$, as $\left.\left.\Gamma \cap B_{\frac{r_{j}}{4}}(0)=r_{j} \partial \Omega\left(\left(u_{1}\right)_{r_{j}}\right)\right) \cap B_{\frac{1}{4}}(0)\right)$. Let us prove that the set of regular points is open in $\Gamma$.

By the elliptic regularity theory, see Corollary 1.8 in [17], $u_{1} \in C^{1, \alpha}\left(\overline{\Omega\left(u_{1}\right)} \cap B_{\frac{r_{j}}{8}}(0)\right)$ and $u_{2} \in C^{1, \alpha}\left(\overline{\Omega\left(u_{2}\right)} \cap B_{\frac{r_{j}}{8}}(0)\right)$, thus

$$
u(x)=\frac{\partial u_{1}}{\partial \nu_{1}}(0)<x, \nu>^{+}-\frac{\partial u_{2}}{\partial \nu_{2}}(0)<x, \nu>^{-}+o(|x|),
$$

and by Theorem 7.4

$$
\frac{\partial u_{1}}{\partial \nu_{1}}(0)=\frac{\partial u_{2}}{\partial \nu_{2}}(0)>0
$$


where $\nu_{i}$ is the interior unit normal vector to $\Omega\left(u_{i}\right)$. In particular, $u$ has the asymptotic behavior (3.1) at 0 . By the $C^{1, \alpha}$ local regularity of $u_{1}$ and $u_{2}$ up to the free boundary, there exists $s<r_{j} / 8$, such that:

$$
\frac{\partial u_{1}}{\partial \nu_{1}}\left(x_{0}\right)>0, \quad \frac{\partial u_{2}}{\partial \nu_{2}}\left(x_{0}\right)>0, \text { for any } x_{0} \in \Gamma \cap B_{s}(0),
$$

and

$$
\left.\left.u(x)=\frac{\partial u_{1}}{\partial \nu_{1}}\left(x_{0}\right)<x-x_{0}, \nu\right\rangle^{+}-\frac{\partial u_{2}}{\partial \nu_{2}}\left(x_{0}\right)<x-x_{0}, \nu\right\rangle^{-}+o\left(\left|x-x_{0}\right|\right) .
$$

Hence each $x_{0} \in \Gamma \cap B_{s}(0)$ is a regular point of $u$. Actually, again from Theorem 7.4, we have that $\frac{\partial u_{1}}{\partial \nu_{1}}\left(x_{0}\right)=\frac{\partial u_{2}}{\partial \nu_{2}}\left(x_{0}\right)$. We have proven that the set of regular points is an open set of $\Gamma$, locally of class $C^{1, \alpha}$ and this concludes the proof of the theorem.

\section{ApPEndix}

Lemma 9.1. Assume $r, \gamma, c>0$, and let

$$
\psi(x)=c\left(\frac{r^{\gamma}}{|x|^{\gamma}}-1\right), \quad x \neq 0 .
$$

Then, the following holds.

i) $\psi(x)>0$ if $|x|<r, \psi(x)=0$ if $|x|=r, \psi(x)<0$ if $|x|>r$.

ii) If $\nu$ is the interior normal unit vector of $B_{r}(0)$, then

iii) For any $x \in B_{r}(0)$,

$$
\nabla \psi(x)=\frac{c \gamma}{r} \nu \quad \text { for any } x \in \partial B_{r}(0) .
$$

$$
\psi(x) \geq \frac{c \gamma}{r}(r-|x|) .
$$

iv) If $\gamma=\frac{\Lambda(n-1)-\lambda}{\lambda}$, then $\mathcal{M}^{-}(\psi)(x)=0$ for all $x \neq 0$.

Proof. Property (i) is immediate.

To prove (ii)-(iv), let us compute the gradient and the Hessian matrix of $\psi$. We get, for $x \neq 0$,

and

$$
\nabla \psi(x)=-c \gamma r^{\gamma} \frac{x}{|x|^{\gamma+2}}
$$

$$
D^{2} \psi(x)=\frac{c \gamma r^{\gamma}}{|x|^{\gamma+2}}\left((\gamma+2) \frac{x \otimes x}{|x|^{2}}-I_{n}\right)
$$

where $I_{n}$ is the $n \times n$ identity matrix.

In particular, if $|x|=r$ and $\nu=-\frac{x}{r}$ is the interior normal unit vector of $B_{r}(0)$ at $x$, then we see that

which proves (ii).

$$
\nabla \psi(x)=-\frac{c \gamma}{r} \frac{x}{r}=\frac{c \gamma}{r} \nu
$$

To prove (iii), let us denote $\rho=|x|$ and let $\psi(\rho)=c\left(\frac{r^{\gamma}}{\rho^{\gamma}}-1\right)$. Then using that $\psi^{\prime}(r)=-\frac{c \gamma}{r}$ and that $\psi^{\prime \prime}(\rho) \geq 0$, we get

which gives (iii).

$$
\psi(\rho) \geq \frac{c \gamma}{r}(r-\rho)
$$


Next, it is easy to see that, given any $n \times n$-matrix $A$ with eigenvalues $\lambda_{1}, \ldots, \lambda_{n}$, then the eigenvalues of $A-I_{n}$ are $\lambda_{1}-1, \ldots, \lambda_{n}-1$. Therefore, since the eigenvalues of $\frac{x \otimes x}{|x|^{2}}$ are $\lambda_{1}=\ldots=\lambda_{n-1}=0$ and $\lambda_{n}=1$, we infer that $(\gamma+2) \frac{x \otimes x}{|x|^{2}}-I_{n}$ has $(n-1)$ negative eigenvalues equal to -1 and one positive eigenvalue equal to $(\gamma+1)$. In particular

$$
\mathcal{M}^{-}(\psi)=\frac{c \gamma r^{\gamma}}{|x|^{\gamma+2}}[\lambda(\gamma+1)-\Lambda(n-1)]
$$

Property (iv) then follows.

Lemma 9.2. Let $\phi$ be the solution of

$$
\begin{cases}\mathcal{F}^{-}(\phi)=0 & \text { in } B_{r}(0) \backslash B_{\frac{r}{2}}(0) \\ \phi=1 & \text { on } \partial B_{\frac{r}{2}}(0) \\ \phi=0 & \text { on } \partial B_{r}(0) .\end{cases}
$$

Then, $\phi=\phi(|x|)$ is a radial function and there exists a constant $\sigma>0$ independent of $r$ such that for $x \in B_{r}(0) \backslash B_{\frac{r}{2}}(0)$ and $y_{0} \in \partial B_{r}(0)$,

$$
\phi(x)=\frac{\sigma}{r}<x-y_{0}, \nu>+o\left(\left|x-y_{0}\right|\right),
$$

where $\nu$ is the interior normal unit vector of $B_{r}(0)$ at $y_{0}$.

Proof. Let $\varphi$ be the solution of (9.1) with $r=1$. Then, since $\mathcal{F}^{-}$is a concave operator, we have that $\varphi \in C^{2, \alpha}\left(\overline{B_{1}(0)} \backslash B_{\frac{r}{2}}(0)\right)$, see [2]. Let $O$ be any orthogonal matrix and let $v(x):=\varphi(O x)$. By Proposition 2.2, $\mathcal{F}^{-}$is invariant under rotations, thus $v$ is solution of (9.1) and by uniqueness, $\varphi(O x)=\varphi(x)$. Since the latter equality holds true for any orthogonal matrix $O$, we infer that $\varphi$ is a radial function, $\varphi=\varphi(|x|)$.

Let $\psi_{1}(x):=1 /\left(2^{\gamma}-1\right)\left(\frac{1}{|x|^{\gamma}}-1\right)$ where $\gamma=\frac{\Lambda(n-1)-\lambda}{\lambda}$, and let $\psi_{2}$ be the harmonic function solution of

$$
\begin{cases}\Delta \psi_{2}=0 & \text { in } B_{1}(0) \backslash B_{\frac{1}{2}}(0) \\ \psi_{2}=1 & \text { on } \partial B_{\frac{1}{2}}(0) \\ \psi_{2}=0 & \text { on } \partial B_{1}(0)\end{cases}
$$

i.e., for $n>2, \psi_{2}(x)=1 /\left(2^{n-2}-1\right)\left(\frac{1}{|x|^{n-2}}-1\right)$. Then by Lemma 9.1 and the comparison principle, for $x \in B_{1}(0) \backslash B_{\frac{1}{2}}(0)$,

$$
\psi_{1}(x) \leq \varphi(x) \leq \psi_{2}(x)
$$

and thus there exists $\sigma, \gamma /\left(2^{\gamma}-1\right) \leq \sigma \leq(n-2) /\left(2^{n-2}-1\right)$, such that if $y_{0} \in \partial B_{1}(0)$,

$$
\varphi(x)=\sigma<x-y_{0}, \nu>+o\left(\left|x-y_{0}\right|\right) .
$$

The lemma is proven by noticing that $\phi(x)=\varphi(x / r)$ is the solution of (9.1).

\section{REFERENCES}

[1] R. Argiolas and F. Ferrari. Flat free boundaries regularity in two-phase problems for a class of fully nonlinear elliptic operators with variable coefficients. Interfaces Free Bound., 11(2), 2009.

[2] L. Caffarelli and X. Cabré. Fully nonlinear elliptic equations, volume 43. American Mathematical Society, 1995.

[3] L. Caffarelli and S. Salsa. A geometric approach to free boundary problems, volume 68. American mathematical society Providence, RI, 2005. 
[4] Luis A Caffarelli. A harnack inequality approach to the regularity of free boundaries. Communications on Pure and Applied Mathematics, 39(S1), 1986.

[5] Luis A Caffarelli. A harnack inequality approach to the regularity of free boundaries. part i: Lipschitz free boundaries are $C^{1, \alpha}$. Revista Matemática Iberoamericana, 3(2):139-162, 1987.

[6] Luis A Caffarelli. A harnack inequality approach to the regularity of free boundaries part ii: Flat free boundaries are lipschitz. Communications on Pure and Applied Mathematics, 42(1):55-78, 1989.

[7] Gui-Qiang Chen, Giovanni Comi, and Monica Torres. The Gauss-Green formula for $\mathcal{D} \mathcal{M}^{p}$-fields on open sets. Preprint, 2018.

[8] Gui-Qiang Chen, Monica Torres, and William Ziemer. Gauss-Green theorem for weakly differentiable vector fields, sets of finite perimeter, and balance laws. Communications on Pure and Applied Mathematics, LVII:0242-0304, 2009.

[9] Michael G Crandall, Hitoshi Ishii, and Pierre-Louis Lions. User's guide to viscosity solutions of second order partial differential equations. Bulletin of the American Mathematical Society, 27(1):1-67, 1992.

[10] D. De Silva, F. Ferrari, and S. Salsa. Free boundary regularity for fully nonlinear non-homogeneous two-phase problems. J. Math. Pures Appl. (9), 103(3):658-694, 2015.

[11] M. Feldman. Regularity of lipschitz free boundaries in two-phase problems for fully nonlinear elliptic equations. Indiana Univ. Math. J., 50:1171-1200, 2001.

[12] D. Gilbarg and N.S. Trudinger. Elliptic partial differential equations of second order, volume 224. Springer Verlag, 2001.

[13] Q. Han and F-H Lin. Elliptic partial differential equations, volume 1. American Mathematical Soc., 2011.

[14] H. Ishii and P.-L. Lions. Viscosity solutions of fully nonlinear second-order elliptic partial differential equations. J. Differential Equations, 83(1):26-78, 1990.

[15] H. Jiang and F-H Lin. Zero set of sobolev functions with negative power of integrability. Chinese Annals of Mathematics, 25(01):65-72, 2004.

[16] Nikolaj Vladimirovič Krylov. Controlled diffusion processes, volume 14. Springer Science \& Business Media, 2008.

[17] F. Ma and L. Wang. Boundary first order derivative estimates for fully nonlinear elliptic equations. J. Differential Equations, 252(2):988-1002, 2012.

[18] A. Petrosyan, H. Shahgholian, and N. Uraltseva. Regularity of free boundaries in obstacle-type problems, volume 136. American Mathematical Society Providence (RI), 2012.

[19] V. Quitalo. A free boundary problem arising from segregation of populations with high competition. Archive for Rational Mechanics and Analysis, 210(3):857-908, 2013.

[20] P-Y. Wang. Regularity of free boundaries of two-phase problems for fully nonlinear elliptic equations of second order i. lipschitz free boundaries are $C^{1, \alpha}$. Communications on Pure and Applied Mathematics, 53(7):799-810, 2000.

[21] P-Y. Wang. Regularity of free boundaries of two-phase problems for fully nonlinear elliptic equations of second order. ii. flat free boundaries are lipschitz. Communications in Partial Differential Equations, 27(78):1497-1514, 2002.

[22] P-Y. Wang. Existence of solutions of two-phase free boundary problems for fully nonlinear elliptic equations of second order. J. Geom. Anal., 13(4):715-738, 2003.

The University of Texas at Austin, Department of Mathematics - RLM 8.100, 2515 Speedway Stop C1200, Austin, TX 78712-1202, US

E-mail address: caffarel@math.utexas.edu

The University of Texas at Austin, Department of Mathematics - RLM 8.100, 2515 Speedway Stop C1200, Austin, TX 78712-1202, US

E-mail address: spatrizi@math.utexas.edu

CMuC, Department of Mathematics, University of Coimbra, 3001-501 Coimbra, Portugal

E-mail address: vquitalo@math.utexas.edu

Purdue University, Department of Mathematics, 150 N. University Street, West Lafayette, IN 47907-2067, US

E-mail address: torresm@math.purdue.edu 